This is a self-archived version of an original article. This version may differ from the original in pagination and typographic details.

Author(s): Pauly, D.; Repin, S.

Title: A posteriori estimates for the stationary Stokes problem in exterior domains

Year: 2019

Version: Accepted version (Final draft)

Copyright: (c) 2020 American mathematical society

Rights: $C C B Y-N C-N D 4.0$

Rights url: https://creativecommons.org/licenses/by-nc-nd/4.0/

Please cite the original version:

Pauly, D., \& Repin, S. (2019). A posteriori estimates for the stationary Stokes problem in exterior domains. St. Petersburg Mathematical Journal, 31(3), Article 3.

https://doi.org/10.1090/spmj/1613 


\title{
A POSTERIORI ESTIMATES FOR THE STATIONARY STOKES PROBLEM IN EXTERIOR DOMAINS
}

\author{
D. PAULY AND S. REPIN
}

Dedicated to the memory of S. G. Mikhlin

\begin{abstract}
This paper is concerned with the analysis of the inf-sup condition arising in the stationary Stokes problem in exterior domains and applications to the derivation of computable bounds for the distance between the exact solution of the exterior Stokes problem and a certain approximation (which may be of a rather general form). In the first part, guaranteed bounds are deduced for the constant in the stability lemma associated with the exterior domain. These bounds depend only on known constants and the stability constant related to bounded domains that arise after suitable truncations of the unbounded domains. The lemma in question implies computable estimates of the distance to the set of divergence free fields defined in exterior domains. Such estimates are crucial for the derivation of computable majorants of the difference between the exact solution of the Stokes problem in exterior domains and an approximation from the admissible (energy) class of functions satisfying the Dirichlet boundary condition but not necessarily divergence free (solenoidal). Estimates of this type are often called a posteriori estimates of functional type. The constant in the stability lemma (or equivalently in the inf-sup or LBB condition) serves as a penalty factor at the term that controls violations of the divergence free condition. In the last part of the paper, similar estimates are deduced for the distance to the exact solution for nonconforming approximations, i.e., for those that may violate some continuity and boundary conditions. The case where the dimension of the domain equals 2 requires a special consideration because the corresponding weighted spaces differ from those natural for the dimension 3 (or larger). This special case is briefly discussed at the end of the paper where similar estimates are deduced for the distance to the exact solution of the exterior Stokes problem.
\end{abstract}

\section{§1. INTRODUCTION}

1.1. Notation and nomenclature. Throughout the paper we consider domains in $\mathbb{R}^{d}$, $d \geq 2$, with Lipschitz boundaries. The symbol $\omega$ is used for bounded domains and the boundary of such a domain is denoted by $\gamma$ (typically, the latter is composed of two open and disjoint parts $\gamma_{D}$ and $\gamma_{N}$ associated with the Dirichlet and Neumann parts). Exterior domains (i.e., those having the form $\mathbb{R}^{d} \backslash \bar{\omega}$ ) are denoted by $\Omega$. By the letter $\mathcal{D}$, we denote domains which may be bounded or unbounded depending on the context (if this property is not necessary to outline).

For Lebesgue and Sobolev spaces of functions (scalar, vector, or tensor valued) with generalised square integrable derivatives of the first order we use the standard notation $\mathrm{L}^{2}(\omega)$ and $\mathrm{H}^{1}(\omega)$ (or $\mathrm{L}^{2}(\Omega)$ and $\mathrm{H}^{1}(\Omega)$ ), respectively. The standard inner product, norm, and orthogonality in $\mathrm{L}^{2}(\omega)$ will be denoted by $\langle\cdot, \cdot\rangle_{0, \omega},\|\cdot\|_{0, \omega}$, and $\perp_{0, \omega}$. If $\gamma_{D} \neq \varnothing$, then the homogeneous Dirichlet boundary conditions are encoded in the space $\mathrm{H}_{\gamma_{D}}^{1}(\mathcal{D})$,

2010 Mathematics Subject Classification. 35J57,65N15,76D07.

Key words and phrases. Stationary Stokes problem, exterior domains, inf-sup condition, a posteriori estimates. 
which is defined as the closure of compactly supported smooth functions vanishing on $\gamma_{D}$ in the norm of $\mathrm{H}^{1}$.

Also, for bounded domains we use spaces with vanishing mean values ${ }^{1}$

$$
\begin{aligned}
& \mathrm{L}_{\perp}^{2}(\omega):=\mathrm{L}^{2}(\omega) \cap \mathbb{R}^{\perp_{0, \omega}}=\left\{\phi \in \mathrm{L}^{2}(\omega):\langle\phi, 1\rangle_{0, \omega}=0\right\}=\left\{\phi \in \mathrm{L}^{2}(\omega): \int_{\omega} \phi=0\right\}, \\
& \mathrm{H}_{\perp}^{1}(\omega):=\mathrm{H}^{1}(\omega) \cap \mathrm{L}_{\perp}^{2}(\omega)=\left\{\phi \in \mathrm{H}^{1}(\omega): \int_{\omega} \phi=0\right\} .
\end{aligned}
$$

To handle the special case of $\gamma_{D}=\varnothing$ using a unified notation, we introduce the space

$$
\mathrm{L}_{\gamma_{D}}^{2}(\omega):=\left\{\begin{array}{lll}
\mathrm{L}^{2}(\omega) & \text { if } \gamma_{D} \neq \gamma, \\
\mathrm{L}_{\perp}^{2}(\omega) & \text { if } \gamma_{D}=\gamma,
\end{array}\right.
$$

and for the case where $\gamma_{D}=\varnothing$ redefine $\mathrm{H}_{\gamma_{D}}^{1}(\omega)$ by setting

$$
\mathrm{H}_{\gamma_{D}}^{1}(\omega)=\mathrm{H}_{\perp}^{1}(\omega)
$$

To further unify our definitions and extend them to exterior domains, we consider a domain (an open and connected set) $\mathcal{D} \subset \mathbb{R}^{d}, d \geq 2$. This domain may be bounded or unbounded. It has a Lipschitz boundary $\mathcal{B}$, which consists of two relatively open and disjoint parts $\mathcal{B}_{D}, \mathcal{B}_{N} \subset \mathcal{B}$ (such that $\overline{\mathcal{B}}=\overline{\mathcal{B}}_{D} \cup \overline{\mathcal{B}}_{N}$ ) associated with Dirichlet and Neumann boundary conditions. As before, we denote the standard Lebesgue and Sobolev spaces by $\mathrm{L}^{2}(\mathcal{D})$ and $\mathrm{H}^{1}(\mathcal{D})$, respectively. If $\mathcal{B}_{D} \neq \varnothing$, we introduce homogeneous Dirichlet boundary conditions in $\mathrm{H}_{\mathcal{B}_{D}}^{1}(\mathcal{D})$ defined as the closure of

$$
\mathrm{C}_{\mathcal{B}_{D}}^{\infty}(\mathcal{D}):=\left\{\left.\phi\right|_{\mathcal{D}}: u \in \mathrm{C}^{\infty}\left(\mathbb{R}^{d}\right), \operatorname{supp} \phi \text { is compact, } \operatorname{dist}\left(\operatorname{supp} \phi, \mathcal{B}_{D}\right)>0\right\}
$$

in $\mathrm{H}^{1}(\mathcal{D})$. As above we utilise the notations $\mathrm{L}_{\mathcal{B}}^{2}(\mathcal{D})=\mathrm{L}_{\perp}^{2}(\mathcal{D}), \mathrm{L}_{\mathcal{B}_{D}}^{2}(\mathcal{D})$, and $\mathrm{H}_{\varnothing}^{1}(\mathcal{D})=$ $\mathrm{H}_{\perp}^{1}(\mathcal{D})$ provided that $\mathcal{D}$ is bounded. Next, we introduce polynomially weighted spaces

$$
\begin{aligned}
\mathrm{L}_{ \pm 1}^{2}(\mathcal{D}) & :=\left\{\phi \in \mathrm{L}_{\text {loc }}^{2}(\mathcal{D}): \rho^{ \pm 1} \phi \in \mathrm{L}^{2}(\mathcal{D})\right\}, \\
\mathrm{H}_{-1}^{1}(\mathcal{D}) & :=\left\{\phi \in \mathrm{L}_{-1}^{2}(\mathcal{D}): \nabla \phi \in \mathrm{L}^{2}(\mathcal{D})\right\},
\end{aligned}
$$

where the weight function $\rho$ is defined by $\rho(r):=\left(1+r^{2}\right)^{1 / 2}$, and $r(x):=|x|$. The inner product, norm, and orthogonality in $\mathrm{L}_{ \pm 1}^{2}(\mathcal{D})$ are denoted by

$$
\langle\cdot, \cdot\rangle_{ \pm 1, \mathcal{D}}:=\left\langle\rho^{ \pm 2} \cdot, \cdot\right\rangle_{0, \mathcal{D}}, \quad\|\cdot\|_{ \pm 1, \mathcal{D}}, \quad \text { and } \perp_{ \pm 1, \mathcal{D}}
$$

respectively. In the case of a bounded domain, there is no difference between the unweighted and weighted spaces (if we mean that the spaces coincide as sets and possess different inner products). However, in analysis of problems in exterior domains a proper selection of weights is important (in $\S 4.6$ devoted to the case of $d=2$ we define the weighted spaces differently). As before, if $\mathcal{B}_{D} \neq \varnothing$, then the homogeneous Dirichlet boundary conditions are encoded in $\mathrm{H}_{-1, \mathcal{B}_{D}}^{1}(\mathcal{D})$, the closure of $\mathrm{C}_{\mathcal{B}_{D}}^{\infty}(\mathcal{D})$ in $\mathrm{H}_{-1}^{1}(\mathcal{D})$. Finally, for the Stokes equations, we introduce spaces of solenoidal fields

$$
\begin{array}{rlrl}
\mathrm{S}(\mathcal{D}) & :=\left\{\varphi \in \mathrm{H}^{1}(\mathcal{D}): \operatorname{div} \varphi=0\right\}, & \mathrm{S}_{\mathcal{B}_{D}}(\mathcal{D}):=\mathrm{H}_{\mathcal{B}_{D}}^{1}(\mathcal{D}) \cap \mathrm{S}(\mathcal{D}), \\
\mathrm{S}_{-1}(\mathcal{D}) & :=\left\{\varphi \in \mathrm{H}_{-1}^{1}(\mathcal{D}): \operatorname{div} \varphi=0\right\}, \quad \mathrm{S}_{-1, \mathcal{B}_{D}}(\mathcal{D}):=\mathrm{H}_{-1, \mathcal{B}_{D}}^{1}(\mathcal{D}) \cap \mathrm{S}_{-1}(\mathcal{D}) .
\end{array}
$$

\footnotetext{
${ }^{1}$ Throughout this paper, we do not express the respective measure in the notation of integrals, so that, e.g., we often use the notation like this:

$$
\int_{\omega} f=\int_{\omega} f d \lambda=\int_{\omega} f d x, \quad \int_{\gamma} f=\int_{\gamma} f d o=\int_{\gamma} f d s .
$$
}


1.2. Stability lemma and the Stokes problem in bounded domains. The classical stationary Stokes problem consists of finding a vector field $u$ (velocity) and a scalar valued function $p$ (pressure) that solve the system

$$
\begin{aligned}
-\nu \Delta u+\nabla p & =f & & \text { in } \omega, \\
\operatorname{div} u & =0 & & \text { in } \omega, \\
u & =u_{D} & & \text { on } \gamma_{D}, \\
\sigma n & =0 & & \text { on } \gamma_{N},
\end{aligned}
$$

where $\sigma:=\nu \nabla u-p \mathbb{I}, \nu$ (viscosity) is a positive constant or a positive function taking values in $\left[\nu_{\ominus}, \nu_{\oplus}\right], \nu_{\ominus}, \nu_{\oplus}>0$, and $f \in \mathrm{L}^{2}(\omega)$. The boundary conditions are defined by the vector valued function $u_{D}$. Henceforth, we assume that $u_{D}$ is given by a solenoidal vector field $u_{D}$, i.e., the Dirichlet boundary condition is defined by $u_{D} \in \mathrm{S}(\omega)$ in the sense that $u=u_{D}$ on $\gamma_{D}$, i.e.,

$$
u-u_{D} \in \mathrm{H}_{\gamma_{D}}^{1}(\omega) .
$$

If $\gamma=\gamma_{D}$, then we additionally assume that

$$
\int_{\gamma} n \cdot u_{D}=\int_{\omega} \operatorname{div} u_{D}=\left\langle u_{D}, 1\right\rangle_{0, \omega}=0 .
$$

The existence of the corresponding generalised solution follows from the well-known solution theory for uniformly elliptic linear equations and the stability lemma, which plays an important role in the theory of incompressible flows.

Lemma 1.1 (stability lemma, $[22,1,3,13,14]$ ). There exists $c>0$ such that for any $g \in \mathrm{L}_{\gamma_{D}}^{2}(\omega)$ there is a vector field $u_{g} \in \mathrm{H}_{\gamma_{D}}^{1}(\omega)$ with

$$
\operatorname{div} u_{g}=g \quad \text { and } \quad\left\|\nabla u_{g}\right\|_{0, \omega} \leq c\|g\|_{0, \omega} .
$$

Henceforth, the best constants in (6) and similar inequalities for unbounded domains are denoted by the letter $\kappa$, i.e., $\kappa\left(\omega, \gamma_{D}\right)$ is the smallest $c$ in (6). For $u \in \mathrm{H}_{\gamma_{D}}^{1}(\omega)$ we also have the Friedrichs/Poincaré inequality

$$
\|u\|_{0, \omega} \leq c\|\nabla u\|_{0, \omega}
$$

and $c_{\mathrm{FP}}\left(\omega, \gamma_{D}\right)$ denotes the best constant $c$. Hence from Lemma 1.1, we conclude that $u_{g}$ satisfies the inequalities

$$
\frac{1}{c_{\mathrm{FP}}\left(\omega, \gamma_{D}\right)}\left\|u_{g}\right\|_{0, \omega} \leq\left\|\nabla u_{g}\right\|_{0, \omega} \leq \kappa\left(\omega, \gamma_{D}\right)\left\|\operatorname{div} u_{g}\right\|_{0, \omega} .
$$

We notice that in the theory of electrodynamics the function $u_{g}$ is called a regular potential as it admits (for Maxwell's equations) an unphysical (high) regularity and boundary condition, which is much stronger than the usual normal boundary condition related to the divergence operator.

Lemma 1.1 yields several important corollaries. First, it guarantees the solvability of the stationary Stokes problem (in the velocity-pressure posing). By setting $g=\operatorname{div} u_{g}$, Lemma 1.1 immediately yields the well-known inf-sup (or LBB) condition:

$$
\inf _{g \in \mathrm{L}_{\gamma_{D}}^{2}(\omega)} \sup _{u \in \mathrm{H}_{\gamma_{D}}^{1}(\omega)} \frac{\langle g, \operatorname{div} u\rangle_{0, \omega}}{\|g\|_{0, \omega}\|\nabla u\|_{0, \omega}} \geq \frac{1}{\kappa\left(\omega, \gamma_{D}\right)}=: c_{\mathrm{LBB}}
$$

Another direct corollary to Lemma 1.1 is an estimate of the distance between a vector field $u \in \mathrm{H}_{\gamma_{D}}^{1}(\omega)$ and the set $\mathrm{S}_{\gamma_{D}}(\omega)$ (see $[35,36]$ ),

$$
\operatorname{dist}\left(u, \mathrm{~S}_{\gamma_{D}}(\omega)\right):=\inf _{v \in \mathrm{S}_{\gamma_{D}}(\omega)}\|\nabla(u-v)\|_{0, \omega} .
$$


Corollary 1.2. For any $u \in \mathrm{H}_{\gamma_{D}}^{1}(\omega)$ there exists $u_{0} \in \mathrm{S}_{\gamma_{D}}(\omega)$ such that

$$
\operatorname{dist}\left(u, \mathrm{~S}_{\gamma_{D}}(\omega)\right) \leq\left\|\nabla\left(u-u_{0}\right)\right\|_{0, \omega} \leq \kappa\left(\omega, \gamma_{D}\right)\|\operatorname{div} u\|_{0, \omega} .
$$

Proof. For $u \in \mathrm{H}_{\gamma_{D}}^{1}(\omega)$, solve the equation

$$
\operatorname{div} \widetilde{u}=\operatorname{div} u \in \mathrm{L}_{\gamma_{D}}^{2}(\omega)
$$

with $\widetilde{u} \in \mathrm{H}_{\gamma_{D}}^{1}(\omega)$ and the stability estimate

$$
\|\nabla \widetilde{u}\|_{0, \omega} \leq \kappa\left(\omega, \gamma_{D}\right)\|\operatorname{div} u\|_{0, \omega}
$$

by Lemma 1.1. Note that for $\gamma_{D}=\gamma$ we have

$$
\int_{\gamma} n \cdot u=\int_{\omega} \operatorname{div} u=\langle u, 1\rangle_{0, \omega}=0 .
$$

Then

$$
u_{0}:=u-\widetilde{u} \in \mathrm{S}_{\gamma_{D}}(\omega) \text { and }\left\|\nabla\left(u-u_{0}\right)\right\|_{0, \omega}=\|\nabla \widetilde{u}\|_{0, \omega} \leq \kappa\left(\omega, \gamma_{D}\right)\|\operatorname{div} u\|_{0, \omega} .
$$

In $[38,39,40]$, this result was extended to vector fields satisfying nonhomogeneous Dirichlet boundary conditions (and also for vector fields in $\mathrm{W}^{1, q}(\Omega)$ for $q \in(1, \infty)$ ) provided that such a vector field $u$ satisfies $\operatorname{div} u \in \mathrm{L}_{\gamma_{D}}^{2}(\Omega)$, i.e., the mean value condition (8), if $\gamma_{D}=\gamma$. Moreover, it was shown that if the mean value conditions hold true for a collection of subdomains whose union of closures coincides with the closure of $\omega$, then estimates of the distance can be based on local constants associated with subdomains. In the case of nonhomogeneous boundary conditions, a modified version of Corollary 1.2 reads as follows.

Corollary 1.3. For any $u \in \mathrm{H}^{1}(\omega)$ with $\operatorname{div} u \in \mathrm{L}_{\gamma_{D}}^{2}(\omega)$ there exists a solenoidal $u_{0} \in$ $\mathrm{S}(\omega)$ such that $u_{0}-u \in \mathrm{H}_{\gamma_{D}}^{1}(\omega)$, i.e., $\left.u_{0}\right|_{\gamma_{D}}=\left.u\right|_{\gamma_{D}}$, and

$$
\left\|\nabla\left(u_{0}-u\right)\right\|_{0, \omega} \leq \kappa\left(\omega, \gamma_{D}\right)\|\operatorname{div} u\|_{0, \omega} .
$$

It should be noted that Corollary 1.3 can be also viewed as a lifting lemma, because a boundary datum $\left.u\right|_{\gamma_{D}}$ is lifted to the domain $\omega$. In this case lifting is performed with the help of a solenoidal representative.

Estimates of the constant $\kappa\left(\omega, \gamma_{D}\right)$ have been studied in $[10,25,29,44,6]$ and some other publications. It is not difficult to see that the constant $c_{\mathrm{LBB}}$ in $(7)$ is nonnegative and cannot exceed 1 so that $\kappa\left(\omega, \gamma_{D}\right) \geq 1$. It is known that $c_{\mathrm{LBB}}>0$ for any bounded Lipschitz domain (e.g., $c_{\mathrm{LBB}}=1 / \sqrt{d}$ for a ball in $\mathbb{R}^{d}$ ). However, the exact values of this constant are unknown except some for very special cases (for example, we do not know the constant even for a cube!). In [6], simply computable and sufficiently accurate estimates of the constant were obtained for domains in $\mathbb{R}^{2}$ that are included in a ball of radius $R$ and are star-shaped with respect to a concentric ball of radius $\rho$. It was shown that

$$
\kappa(\omega, \gamma) \leq \frac{\sqrt{2}}{\zeta}\left(1+\sqrt{1-\zeta^{2}}\right)^{1 / 2}
$$

where $\zeta=\rho / R$. For $d=3$, estimates of $c_{\mathrm{LBB}}$ are known only for domains with sufficiently regular boundaries (see [29]). A systematic numerical analysis of constants in the infsup condition (7) was performed in [11], where approximate values of the constants were computed for a wide collection of bounded domains. Computational approaches to the evaluation of the distance to the set of divergence free fields based on domain decomposition were suggested in $[38,39,40]$. In our subsequent analysis, we assume that, using the results and methods mentioned above, we are able to find a majorant of 
the constant $\kappa\left(\omega, \gamma_{D}\right)$ for bounded domains $\omega$ that arise as truncations of an unbounded domain $\Omega$.

1.3. A posteriori estimates. Estimates of the distance to $S_{\gamma_{D}}(\omega)$ are not merely of theoretical value. They are important for the quantitative analysis of boundary value problems generated by incompressible media models (e.g., in the theory of viscous incompressible fluids). First, estimates of this type are necessary for getting computable bounds for the difference between the exact solution of a boundary value problem and an approximation obtained by some computational procedure. The term "computable" means that the corresponding estimates do not involve unknown functions and constants and can indeed be computed by means of an approximate solution only. Estimates of this type are often called a posteriori error estimates and nowadays are widely used in the quantitative analysis of mathematical problems. Unlike the a priori (asymptotic) convergence estimates, a posteriori estimates provide an explicit verification of the accuracy of a particular numerical solution. First methods of a posteriori error control for PDEs originates from the works of W. Prager and J. L. Synge [33] and S. G. Mikhlin [19]. The latter monograph contains a pioneering idea of a new approach to error estimation, which differs principally from asymptotic rate convergence estimates dominated at that time and several decades subsequently. For variational problems generated by quadratic type functionals

$$
J(v)=\frac{1}{2} a(v, v)-\langle f, v\rangle, \quad f \in V,
$$

where $V$ is a Hilbert space and $a: V \times V \rightarrow \mathbb{R}$ is a $V$-elliptic bilinear form, S. Mikhlin deduced the principal relation

$$
\frac{1}{2} a(u-v, u-v)=J(v)-J(u) .
$$

Here $u$ is the minimizer that satisfies

$$
J(u)=\min _{w \in V} J(w) \quad \text { and } \quad v \in V
$$

is any function compared with $u$. Since the exact infimum is unknown, it is impossible to use (11) directly. In [19], it was suggested to estimate $J(u)$ from below using a dual variational problem and further apply the orthogonal projection method of $\mathrm{H}$. Weyl [48]. Certainly, these first estimates were derived for a rather limited set of problems and suffered from serious restrictions imposed on the set of functions that are admissible in the dual setting. For these reasons, they were rarely used in computational practice. Moreover, the methods developed in 1970-1980 for measuring errors of finite element approximations (such as the "gradient averaging" and "residual" methods, see, e.g., [47] and the references therein) were based on different grounds. These methods strongly exploit properties of a particular approximation computed on a particular mesh. In essence, they provide certain error indicators (for mesh adaptive procedures) rather than guaranteed error bounds. Subsequent studies focused on the problem of guaranteed error control (performed in the 1990s) confirmed the idea (encompassed in (11)) that the corresponding methods should be justified on the functional level by means of the same mathematical tools that are used in analysis of PDEs without attracting specific features of approximations and numerical methods. If we have a general (universal) estimate of the distance between a function and the exact solution of a boundary value problem, then it can be used with any approximation and requires no changes if one approximation (mesh) is replaced by another. In the last two decades computable bounds of this type has been derived and tested for a wide spectrum of problems (see [34, 23, 37, 17] and many other publications cited in these monographs). For clear reasons, they are often called 
a posteriori estimates of functional type. They differ from others due to two important properties: the estimates

(a) do not contain constants associated with a particular finite dimensional subspace (mesh) and a method used to solve the problem and

(b) are valid for any approximation in the energy space and do not use special conditions required for the exact solution (e.g., extra regularity) or its approximation (e.g., Galerkin orthogonality, quasi-uniformity of meshes).

A posteriori estimates of functional type involve only global constants generated by functional inequalities such as various embedding estimates, trace inequalities, Poincaré, Maxwell, Korn inequalities, etc. It should be noted that although constants of this type do not appear in (11) (and in the estimates derived in [33]), the importance of studying them was already understood by Mikhlin (see [20]). The reader can find an overview of the history of a posteriori error estimation methods and a subsequent exposition of the functional approach to the problem in [37].

First estimates of the distance between a function in the energy space and the exact solution of the stationary Stokes problem in a bounded domain were derived in [35] (by means of the variational duality method) and in [36] (by transformations of the integral identity that defines the corresponding weak solution). It is worth starting a short overview of these results with the error identity

$$
\nu\|\nabla(v-u)\|^{2}+\nu^{-1}\left\|\tau_{f}-\sigma\right\|^{2}=2\left(I(v)-I^{*}\left(\tau_{f}\right)\right),
$$

which can be viewed as an analog of (11) for the stationary Stokes problem. Here $v \in \mathrm{S}_{\gamma}(\omega)$ and

$$
\tau_{f} \in \mathrm{L}_{f}^{2}(\omega):=\left\{\tau \in \mathrm{L}^{2}(\omega):\langle\tau, \nabla w\rangle_{0, \omega}=\langle f, w\rangle_{0, \omega} \quad \forall w \in \mathrm{S}_{\gamma}(\omega)\right\}
$$

are regarded as approximations of the exact velocity field $u$ and exact stress fiels $\sigma$, respectively. Identity (12) is fulfilled for any $v \in \mathrm{S}_{\gamma}(\omega)$ and any $\tau_{f} \in \mathrm{L}_{f}^{2}(\omega)$. However, it is not very useful for practice for the same reasons as (11), namely, the functions in $\mathrm{S}_{\gamma}(\omega)$ and $\mathrm{L}_{f}^{2}(\omega)$ are subject to differential relations. In [35] (see also [37]), a way was shown to overcome these difficulties by using computable estimates of distances to the sets $\mathrm{S}_{\gamma}(\omega)$ and $\mathrm{L}_{f}^{2}(\omega)$. As a result, the following estimates for the velocity and pressure fields were derived:

$$
\begin{gathered}
\nu\|\nabla(u-\widetilde{u})\|_{0, \omega} \leq\|\tau+\widetilde{p} \mathbb{I}-\nu \nabla \widetilde{u}\|_{0, \omega}+c_{\mathrm{FP}}(\omega, \gamma)\|\operatorname{div} \tau+f\|_{0, \omega} \\
+2 \nu \kappa(\omega, \gamma)\|\operatorname{div} \widetilde{u}\|_{0, \omega}, \\
\frac{1}{2 \kappa(\omega, \gamma)}\|p-\widetilde{p}\|_{0, \omega} \leq\|\tau+\widetilde{p} \mathbb{I}-\nu \nabla \widetilde{u}\|_{0, \omega}+c_{\mathrm{FP}}(\omega, \gamma)\|\operatorname{div} \tau+f\|_{0, \omega} \\
+\nu \kappa(\omega, \gamma)\|\operatorname{div} \widetilde{u}\|_{0, \omega} .
\end{gathered}
$$

Here $\nu$ is a positive constant and $\widetilde{u} \in \mathrm{H}^{1}(\omega)$ is a vector-valued function satisfying the Dirichlet boundary conditions. The function $\widetilde{u}$ is regarded as an approximation of the exact velocity $u$. Similarly, $\widetilde{p}$ is a square integrable function (with zero mean value if the Dirichlet conditions are imposed on the whole boundary $\gamma$ ) viewed as an approximation of $p$ and $\tau \in \mathrm{L}^{2}(\omega)$ is an approximation of the exact stress field $\sigma$. The right-hand sides of (13) and (14) have a clear meaning: they contain three nonnegative terms that vanish if the approximations coincide with the exact velocity, pressure, and stress, respectively. In other cases, the terms can be viewed as penalties for possible violations of the three basic relations that form (1) and (2).

It is easy to see that the constant $\kappa(\omega, \gamma)$ plays an important role in (13) and (14) and, therefore, it is indeed necessary to have guaranteed majorants of this constant. 
These constants arise in the a posteriori analysis of a numerical solution if it satisfies the divergence free condition only approximately. If the constant $\kappa\left(\omega, \gamma_{D}\right)$ is known, then by using Corollary 1.2 we can deduce guaranteed and fully computable error estimates. For problems in bounded Lipschitz domains the respective results were presented in $[35,36,37,39]$ and other publications cited therein. Also, it should be mentioned that explicit bounds of the constant $\kappa(\omega, \gamma)$ are required not only for Stokes type problems. They arise in other continuous media problems, which are not related to viscous fluids (e.g., see [42]).

Subsequently analogous estimates for the velocity and pressure fields were obtained for the Stokes problem in the velocity-vorticity-pressure formulation [18] and for the generalised Stokes problem [41]. In [7], such estimates were derived for a class of stationary problems associated with nonlinear viscous fluids and in [24] for the evolutionary Stokes problem. We note that the approach used in these publications and in the present paper differs essentially from the so-called residual method often used in the finite element community for getting indicators of approximation errors (see, e.g., [46]).

1.4. Outline of the paper. In the first part of the paper, we recall some known results related to the analysis of boundary value problems in exterior domains, paying a special attention to the stability Lemma 2.3 and the corresponding corollaries.

$\S 3$ is devoted to computable bounds $\kappa_{\oplus}\left(\Omega, \Gamma_{D}\right)$ for the stability constant $\kappa\left(\Omega, \Gamma_{D}\right)$. In Lemma 3.2, we obtain a desired estimate for $\kappa_{\oplus}\left(\Omega, \Gamma_{D}\right)$, which involves known constants and the constant $\kappa\left(\omega, \gamma_{D}\right)$ associated with a bounded domain $\omega$ (which is a suitable truncation of $\Omega$ ). Estimates of the last constant have been derived in several publications cited above, so that we view this problem as solvable (at least in the sense that a certain guaranteed bound of $\kappa\left(\omega, \gamma_{D}\right)$ can be derived). As a result, we obtain estimates of the distance between a vector field in $\Omega$ and the respective set of solenoidal fields defined in $\Omega$ and satisfying the same boundary conditions (Lemma 3.4). These estimates are used in $\S 4$, we derive a posteriori error estimates of functional type, which are valid for a wide class of approximate solutions to the stationary Stokes problem in exterior domains.

Estimates for the velocity are obtained in three different forms. The first (and the simplest) form is valid for approximations in $\mathrm{S}(\Omega)$, i.e., for solenoidal vector-valued functions that satisfy the Dirichlet boundary condition exactly (Theorem 4.1). These estimates do not contain the constant $\kappa\left(\Omega, \Gamma_{D}\right)$. Estimates of the second type are valid for approximations in $\mathrm{H}^{1}(\Omega)$ still satisfying the boundary condition exactly but admit possibly nonsolenoidal functions (Theorem 4.2). They involve a term that penalises possible violation of the solenoidality condition and has the constant $\kappa\left(\Omega, \Gamma_{D}\right)$ as a penalty factor. Finally, the most general form of the estimate is applicable for nonconforming approximations, which even may not belong to the energy class $\mathrm{H}^{1}(\Omega)$ (Theorem 4.6). It involves one more term that can be viewed as a measure of the distance to the energy class natural for the velocity function. Also, we deduce estimates for approximations of the pressure (Theorem 4.4 and Theorem 4.6) and the stress field (Subsection 4.4). In $\S 4.5$ we consider lower bounds and in $\S 4.6$ we adapt our results to the special case of space dimension $d=2$.

\section{§2. Preliminaries}

2.1. Exterior domain and main functional inequalities. We consider an exterior domain $\Omega \subset \mathbb{R}^{d}$, where $d \geq 3$ (the special case of $d=2$ is studied in Subsection 4.6), with a (strong) Lipschitz boundary $\Gamma$, which is composed of two open and disjoint parts $\Gamma_{D}, \Gamma_{N} \subset \Gamma$ (Dirichlet and Neumann parts) with $\bar{\Gamma}=\bar{\Gamma}_{D} \cup \bar{\Gamma}_{N}$. Moreover, we assume 
that there exist $0<r_{1}<r_{2}$ such that $\mathbb{R}^{d} \backslash \Omega \subset B_{r_{1}}$ and denote (see Figure 1)

$$
\omega:=\Omega_{r_{2}}:=\Omega \cap B_{r_{2}}, \quad \gamma=\Gamma \cup S_{r_{2}}, \quad \gamma_{D}:=\Gamma_{D} \cup S_{r_{2}},
$$

where $B_{r}$ and $S_{r}$ denote the open ball and the sphere of radius $r$ centered at the origin in $\mathbb{R}^{d}$. By $\eta$ we denote a Lipschitz continuous cut-off function, which vanishes in the ball ${ }^{2} \bar{B}_{r_{1}}$, equals 1 in $\mathbb{R}^{d} \backslash B_{r_{2}}$, and takes values in $[0,1]$.

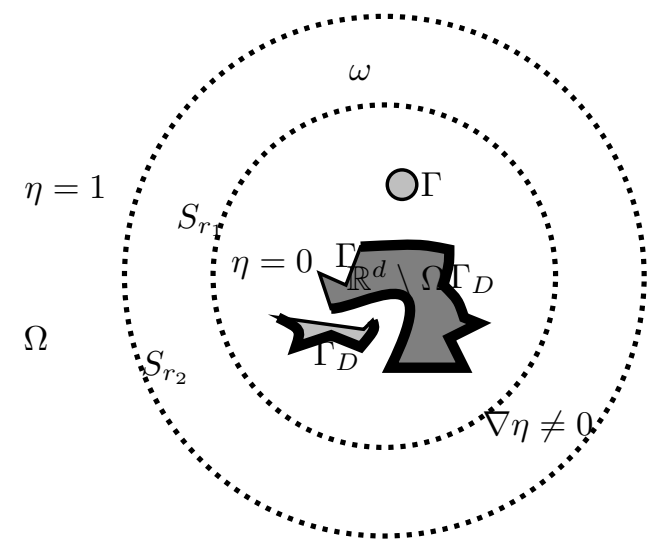

FiguRE $1 . \mathbb{R}^{d} \backslash \bar{\Omega}$ (gray) surrounded by the boundary $\Gamma$ (thin black lines), the boundary part $\Gamma_{D}$ (thick black lines), and the artificial boundary spheres (dashed lines).

The two main ingredients for our proofs are Lemma 1.1 and a few elementary results from the theory of $\nabla$-curl-div-systems in exterior domains and especially $\mathbb{R}^{d}$ (see, e.g., $[16,43]$ or $[12,26]$ and in particular $[28]$ as well as references therein), which can be summarised in the two subsequent lemmas as follows.

Lemma 2.1 (Friedrichs/Poincaré lemma for exterior domains). The following weighted Friedrichs/Poincaré estimates hold true.

(i) There exists $c>0$ such that for all $v \in \mathrm{H}_{-1, \Gamma_{D}}^{1}(\Omega)$ we have

$$
\|v\|_{-1, \Omega} \leq c\|\nabla v\|_{0, \Omega} \text {. }
$$

The best constant $c$ is called the Friedrichs/Poincaré constant and we denote it by $c_{\mathrm{FP}}\left(\Omega, \Gamma_{D}\right)$.

(ii) If $\Gamma_{D}=\Gamma$, then $c_{\mathrm{FP}}(\Omega, \Gamma)=c_{\mathrm{F}}(\Omega)$ (the Friedrichs constant) and

$$
c_{\mathrm{F}}(\Omega) \leq c_{d}:=\frac{2}{d-2} .
$$

Hence for all $v \in \mathrm{H}_{-1, \Gamma}^{1}(\Omega)$ the Friedrichs estimate $\|v\|_{-1, \Omega} \leq c_{d}\|\nabla v\|_{0, \Omega}$ holds true. If $\Gamma_{D}=\varnothing$, then $c_{\mathrm{FP}}(\Omega, \varnothing)$ is replaced by the Poincaré constant $c_{\mathrm{P}}(\Omega)$. In this case, the Poincaré estimate

$$
\|v\|_{-1, \Omega} \leq c_{\mathrm{P}}(\Omega)\|\nabla v\|_{0, \Omega}
$$

is fulfilled for all $v \in \mathrm{H}_{-1}^{1}(\Omega)$.

\footnotetext{
${ }^{2}$ For the sake of simplicity we henceforth operate with the balls $B_{r_{1}}$ and $B_{r_{2}}$. However, if necessary $B_{r_{1}}$ can be replaced by a Lipschitz domain containing $\mathbb{R}^{2} \backslash \bar{\Omega}$ and $B_{r_{2}}$ by another Lipschitz domain containing $B_{r_{1}}$.
} 
(iii) If $\Omega=\mathbb{R}^{d}$, then the Friedrichs and Poincaré constants coincide and, moreover,

$$
c_{\mathrm{FP}}\left(\mathbb{R}^{d}\right)=c_{\mathrm{F}}\left(\mathbb{R}^{d}\right)=c_{\mathrm{P}}\left(\mathbb{R}^{d}\right) \leq c_{d} .
$$

Hence for all $v \in \mathrm{H}_{-1}^{1}\left(\mathbb{R}^{d}\right)$ we have $\|v\|_{-1, \mathbb{R}^{d}} \leq c_{d}\|\nabla v\|_{0, \mathbb{R}^{d}}$.

Note that no boundary or mean value conditions are needed in Lemma 2.1 (because constant functions are not integrable in $\left.\mathrm{L}_{-1}^{2}(\Omega)\right)$.

Lemma 2.2 ( $\nabla$-curl-div lemma for exterior domains). Suppose that $\Omega=\mathbb{R}^{d}$ and $h \in$ $\mathrm{L}^{2}\left(\mathbb{R}^{d}\right)$. Then there exists a unique $v \in \mathrm{H}_{-1}^{1}\left(\mathbb{R}^{d}\right)$ such that $\operatorname{curl} v=0$ and $\operatorname{div} v=h$. Moreover,

$$
\frac{1}{c_{d}}\|v\|_{-1, \mathbb{R}^{d}} \leq\|\nabla v\|_{0, \mathbb{R}^{d}}=\|\operatorname{div} v\|_{0, \mathbb{R}^{d}}=\|h\|_{0, \mathbb{R}^{d}} .
$$

The results of Lemma 2.2 are well known and follow directly by Fourier analysis, see, e.g., $[30,31,32,16]$ or $[12,26]$. Notice that the well-known equation $-\Delta=$ curl $^{*}$ curl $-\nabla$ div implies the identity

$$
\|\nabla v\|_{0, \mathbb{R}^{d}}^{2}=\|\operatorname{curl} v\|_{0, \mathbb{R}^{d}}^{2}+\|\operatorname{div} v\|_{0, \mathbb{R}^{d}}^{2}
$$

for all $v \in \mathrm{C}^{\infty}\left(\mathbb{R}^{d}\right)$ having compact support. By density and continuity arguments it can be extended to all $v \in \mathrm{H}_{-1}^{1}\left(\mathbb{R}^{d}\right)$. Hence the relation

$$
\|\nabla v\|_{0, \mathbb{R}^{d}}=\|\operatorname{div} v\|_{0, \mathbb{R}^{d}}
$$

in Lemma 2.2 follows immediately.

2.2. Stability lemma and Stokes problem in exterior domains. For exterior domains we have a result similar to Lemma 1.1 (see [9]).

Lemma 2.3 (stability lemma for exterior domains). There exists $c>0$ such that for any $h \in \mathrm{L}^{2}(\Omega)$ there is a vector field

$$
u_{h} \in \mathrm{H}_{-1, \Gamma_{D}}^{1}(\Omega)
$$

with

$$
\operatorname{div} u_{h}=h \quad \text { and } \quad\left\|\nabla u_{h}\right\|_{0, \Omega} \leq c\|h\|_{0, \Omega} .
$$

The best constant is denoted by $\kappa\left(\Omega, \Gamma_{D}\right)$.

In the subsequent Lemma 3.2 we present computable upper bounds for $\kappa\left(\Omega, \Gamma_{D}\right)$.

Remark 2.4. In [9] only the case of $\Gamma_{D}=\Gamma$ was considered, but it is trivial that then the stability lemma is true for general $\Gamma_{D}$ as well. It should be noted that unlike the case of a bounded domain, no mean value condition is imposed on $h$ even if $\Gamma_{D}=\Gamma$.

Lemma 2.3 immediately implies the inf-sup condition.

Corollary 2.5 (inf-sup lemma for exterior domains). We have

$$
\inf _{h \in \mathrm{L}^{2}(\Omega)} \sup _{u \in \mathrm{H}_{-1, \Gamma_{D}}^{1}(\Omega)} \frac{\langle h, \operatorname{div} u\rangle_{0, \Omega}}{\|h\|_{0, \Omega}\|\nabla u\|_{0, \Omega}} \geq \frac{1}{\kappa\left(\Omega, \Gamma_{D}\right)} .
$$

From now on, let $f \in \mathrm{L}_{1}^{2}(\Omega), u_{D} \in \mathrm{S}_{-1}(\Omega)$ be given data. The classical Stokes problem in an exterior domain $\Omega$ consists of finding a vector-valued function $u$ (velocity) and a scalar valued function $p$ (pressure) satysfying the system

$$
\begin{aligned}
-\operatorname{div} \sigma & =f & & \text { in } \Omega, \\
\sigma & =\nu \nabla u-p \mathbb{I} & & \text { in } \Omega, \\
\operatorname{div} u & =0 & & \text { in } \Omega, \\
u & =u_{D} & & \text { on } \Gamma_{D}, \\
\sigma n & =0 & & \text { on } \Gamma_{N},
\end{aligned}
$$


where $u$ additionally satisfies a proper decay conditions at infinity, so that $u \in \mathrm{S}_{-1}(\Omega)$ or $u \in \mathrm{H}_{-1}^{1}(\Omega)$, i.e.,

$$
u \in \mathrm{L}_{-1}^{2}(\Omega) \text { and } \nabla u \in \mathrm{L}^{2}(\Omega) .
$$

The classical (pointwise) condition reads

$$
u(x) \stackrel{|x| \rightarrow \infty}{\longrightarrow} 0 .
$$

The corresponding generalised solution

$$
u \in u_{D}+\mathrm{S}_{-1, \Gamma_{D}}(\Omega)
$$

is defined by the integral identity (see, e.g., $[13,9]$ )

$$
\langle\nu \nabla u, \nabla \varphi\rangle_{0, \Omega}=\langle f, \varphi\rangle_{0, \Omega} \quad \forall \varphi \in \mathrm{S}_{-1, \Gamma_{D}}(\Omega) .
$$

Note that, since

$$
\langle f, \varphi\rangle_{0, \Omega}=\left\langle\rho f, \rho^{-1} \varphi\right\rangle_{0, \Omega},
$$

the right-hand side of (23) is well defined. Using the Ansatz $u=u_{D}+\widehat{u}$ with $\widehat{u} \in$ $\mathrm{S}_{-1, \Gamma_{D}}(\Omega)$, we reduce this formulation to find $\widehat{u} \in \mathrm{S}_{-1, \Gamma_{D}}(\Omega)$ such that for all $\varphi \in$ $\mathrm{S}_{-1, \Gamma_{D}}(\Omega)$ we have

$$
\langle\nu \nabla \widehat{u}, \nabla \varphi\rangle_{0, \Omega}=\langle f, \varphi\rangle_{0, \Omega}-\left\langle\nu \nabla u_{D}, \nabla \varphi\right\rangle_{0, \Omega} .
$$

Another formulation taking the pressure into account leads to the following saddle point formulation: find

$$
(u, p) \in\left(u_{D}+\mathrm{H}_{-1, \Gamma_{D}}^{1}(\Omega)\right) \times \mathrm{L}^{2}(\Omega)
$$

such that for all $(\varphi, \phi) \in \mathrm{H}_{-1, \Gamma_{D}}^{1}(\Omega) \times \mathrm{L}^{2}(\Omega)$ we have

$$
\begin{aligned}
\langle\nu \nabla u, \nabla \varphi\rangle_{0, \Omega}-\langle p, \operatorname{div} \varphi\rangle_{0, \Omega} & =\langle f, \varphi\rangle_{0, \Omega}, \\
\langle\operatorname{div} \widehat{u}, \phi\rangle_{0, \Omega} & =0 .
\end{aligned}
$$

By standard arguments (see, e.g., $[8,9,13]$ ) and the inf-sup lemma, we know that for $f \in \mathrm{L}_{1}^{2}(\Omega)$ and $u_{D} \in \mathrm{S}_{-1}(\Omega)$ the Stokes system is uniquely solvable with $u \in u_{D}+$ $\mathrm{S}_{-1, \Gamma_{D}}(\Omega)$ and $p \in \mathrm{L}^{2}(\Omega)$. Moreover, the solution meets the estimates

$$
\begin{aligned}
\nu\|\nabla \widehat{u}\|_{0, \Omega} & \leq c_{\mathrm{FP}}\left(\Omega, \Gamma_{D}\right)\|f\|_{1, \Omega}+\nu\left\|\nabla u_{D}\right\|_{0, \Omega}, \\
\nu\|\nabla u\|_{0, \Omega} & \leq c_{\mathrm{FP}}\left(\Omega, \Gamma_{D}\right)\|f\|_{1, \Omega}+2 \nu\left\|\nabla u_{D}\right\|_{0, \Omega}, \\
\|p\|_{0, \Omega} & \leq 2 \kappa\left(\Omega, \Gamma_{D}\right)\left(c_{\mathrm{FP}}\left(\Omega, \Gamma_{D}\right)\|f\|_{1, \Omega}+\nu\left\|\nabla u_{D}\right\|_{0, \Omega}\right) .
\end{aligned}
$$

\section{$\S 3$. Estimates of $\kappa\left(\Omega, \Gamma_{D}\right)$}

Our goal is to deduce majorants for the constant in Lemma 2.3 that involve only known constants (such as $c_{d}$ ) and stability constants for a bounded domain $\omega$ generated by a certain truncation of $\Omega$.

First we discuss the simplest case of a compactly supported right-hand side.

Lemma 3.1. There exists $c>0$ such that for all $h \in \mathrm{L}^{2}(\Omega)$ with $\operatorname{supp} h \subset \bar{\omega}$ and $h \in \mathrm{L}_{\gamma_{D}}^{2}(\omega)$, i.e., $\int_{\Omega} h=\int_{\omega} h=0$ if $\Gamma_{D}=\Gamma$, there exists a vector field $u_{h} \in \mathrm{H}_{-1, \Gamma_{D}}^{1}(\Omega)$ such that

$$
\operatorname{div} u_{h}=h \quad \text { and } \quad\left\|\nabla u_{h}\right\|_{0, \Omega} \leq c\|h\|_{0, \Omega} .
$$

The function $u_{h}$ can be chosen with compact support in $\bar{\omega}$, in particular,

$$
u_{h} \in \mathrm{H}_{\gamma_{D}}^{1}(\omega) \subset \mathrm{H}_{\Gamma_{D}}^{1}(\Omega) .
$$

In this case, $\kappa\left(\Omega, \Gamma_{D}\right) \leq \kappa\left(\omega, \gamma_{D}\right)$.

Hence we arrive at the rather obvious conclusion that for a compactly supported $h$ the constant $\kappa\left(\Omega, \Gamma_{D}\right)$ can be estimated by $\kappa\left(\omega, \gamma_{D}\right)$. 
Proof of Lemma 3.1. Set

$$
g:=\left.h\right|_{\omega} \in \mathrm{L}_{\gamma_{D}}^{2}(\omega)
$$

By Lemma 1.1 there exist $\kappa\left(\omega, \gamma_{D}\right)>0$ and $u_{g} \in \mathrm{H}_{\gamma_{D}}^{1}(\omega), u_{g}=0$ on $S_{r_{2}}$ such that $\operatorname{div} u_{g}=g$ and $\left\|\nabla u_{g}\right\|_{0, \omega} \leq \kappa\left(\omega, \gamma_{D}\right)\|g\|_{0, \omega}$. Let $u_{h}$ be the extension by zero of $u_{g}$ to $\Omega$. Then $u_{h} \in \mathrm{H}_{\Gamma_{D}}^{1}(\Omega)$ and $\operatorname{supp} u_{h}=\operatorname{supp} u_{g} \subset \bar{\omega}$. Moreover, div $u_{h}=h$ and

$$
\left\|\nabla u_{h}\right\|_{0, \Omega}=\left\|\nabla u_{g}\right\|_{0, \omega} \leq \kappa\left(\omega, \gamma_{D}\right)\|g\|_{0, \omega}=\kappa\left(\omega, \gamma_{D}\right)\|h\|_{0, \Omega},
$$

completing the proof.

Now we present the main result, which provides an upper bound for the best constant $\kappa\left(\Omega, \Gamma_{D}\right)$ in the stability Lemma 2.3 for exterior domains as well as an alternative proof of this lemma.

Lemma 3.2. Let $\Omega$ and $\omega$ be defined by (15). Then

$$
\kappa\left(\Omega, \Gamma_{D}\right) \leq \kappa_{\oplus}\left(\Omega, \Gamma_{D}\right):=(1+\kappa)\left(1+c_{d} \alpha \rho\left(r_{2}\right)\right)
$$

with $\kappa\left(\Omega, \Gamma_{D}\right)$ from Lemma 2.3, where $\alpha$ and $\kappa$ are defined in (29) and (31), i.e.,

$$
\alpha=\alpha\left(r_{1}, r_{2}, \eta\right)=\max _{x \in \bar{B}_{r_{2}} \backslash B_{r_{1}}}|\nabla \eta(x)|, \quad \kappa=\min \left\{\kappa\left(\omega, \gamma_{D}\right), \kappa(\omega, \gamma)\right\} .
$$

Remark 3.3. It should be noted that $\kappa \leq \kappa\left(\omega, \gamma_{D}\right)$ and $\kappa \leq \kappa(\omega, \gamma)$, where the second bound means that $\kappa$ can be estimated independently of the boundary part $\Gamma_{D}$ of $\Gamma$. Moreover, the constant $\kappa_{\oplus}\left(\Omega, \Gamma_{D}\right)$ depends on the dimension $d$, the radii $r_{1}, r_{2}$, and the Lipschitz continuous cut-off function $\eta$. Optimal values of these parameters (which minimise the constant) are not known a priori and should be defined by solving an additional algebraic problem. If $\eta$ is constructed, e.g., by a simple affine function with $r_{2}=r_{1}+1$, then $\alpha=1$ and we have one possible upper bound

$$
\kappa_{\oplus}\left(\Omega, \Gamma_{D}\right) \leq(1+\kappa)\left(1+c_{d} \sqrt{1+r_{2}^{2}}\right) \leq(1+\kappa(\omega, \gamma))\left(1+\frac{2 \sqrt{2}}{d-2} r_{2}\right) .
$$

In some cases, this bound may be rather coarse, but, anyhow, it presents a guaranteed upper bound of the stability constant $\kappa_{\oplus}\left(\Omega, \Gamma_{D}\right)$ associated with the exterior domain $\Omega$.

Proof of Lemma 3.2. We extend $h$ by 0 to $\mathbb{R}^{d} \backslash \bar{\Omega}$ and identify the extended function with $h \in \mathrm{L}^{2}\left(\mathbb{R}^{d}\right)$. By Lemma 2.2 we have a vector field $v \in \mathrm{H}_{-1}^{1}\left(\mathbb{R}^{d}\right)$ with curl $v=0$ and $\operatorname{div} v=h$ in $\mathbb{R}^{d}$ as well as

$$
\frac{1}{c_{d}}\|v\|_{-1, \mathbb{R}^{d}} \leq\|\nabla v\|_{0, \mathbb{R}^{d}}=\|\operatorname{div} v\|_{0, \mathbb{R}^{d}}=\|h\|_{0, \Omega} .
$$

We recall that our cut-off function $\eta$ satisfies $\left.\eta\right|_{B_{r_{1}}}=0$ and $\left.\eta\right|_{\mathbb{R}^{d} \backslash B_{r_{2}}}=1$. Therefore, $\eta v \in \mathrm{H}_{-1, \Gamma}^{1}(\Omega)$ and $\operatorname{supp}(\eta v) \subset \mathbb{R}^{d} \backslash B_{r_{1}}$. In view of Lemma 2.3, our goal is to find a vector-valued function $v_{h} \in \mathrm{H}_{-1, \Gamma_{D}}^{1}(\Omega)$ such that $\operatorname{div} v_{h}=h$ in $\Omega$ and the stability inequality holds true. We suggest to construct it in the form

$$
v_{h}:=\eta v+v_{\omega},
$$

where $v_{\omega} \in \mathrm{H}_{\Gamma_{D}}^{1}(\Omega)$ with $\operatorname{supp} v_{\omega} \subset \bar{\omega}$ is the extension by zero to $\Omega$ of some vector-valued function $u_{\omega} \in \mathrm{H}_{\gamma_{D}}^{1}(\omega)$ that is supported in $\bar{\omega}$ and vanishes on $S_{r_{2}}$.

The relation

$$
h=\operatorname{div} v_{h}=\eta h+\nabla \eta \cdot v+\operatorname{div} v_{\omega} \quad \text { in } \Omega
$$

imposes a condition on $v_{\omega}$ and $u_{\omega}$. Let

$$
g:=(1-\eta) h-\nabla \eta \cdot v \in \mathrm{L}^{2}(\omega) \text { in } \omega .
$$


Notice that $\operatorname{supp}(1-\eta) \subset \bar{B}_{r_{2}}$, supp $\nabla \eta \subset \bar{B}_{r_{2}} \backslash B_{r_{1}}$ and hence supp $g \subset \bar{\omega}$ (if we view $g$ as a function in $\Omega$ ). Since

$$
g=(1-\eta) h+\nabla(1-\eta) \cdot v=\operatorname{div}((1-\eta) v)
$$

by the properties of $\eta$ we have by the Gauss or Stokes theorem

$$
\int_{\omega} g=\int_{\gamma}(1-\eta) n \cdot v=\int_{\Gamma} n \cdot v=-\int_{\mathbb{R}^{d} \backslash \bar{\Omega}} \operatorname{div} v=-\int_{\mathbb{R}^{d} \backslash \bar{\Omega}} h=0 .
$$

Hence $g$ has zero mean value independent of the boundary part $\gamma=\gamma_{D}$, i.e., we always have

$$
\int_{\omega} g=0
$$

Now, the existence of $u_{\omega}=u_{g, \gamma_{D}} \in \mathrm{H}_{\gamma_{D}}^{1}(\omega)$, respectively, $u_{\omega}=u_{g, \gamma} \in \mathrm{H}_{\gamma}^{1}(\omega)$ such that $\operatorname{div} u_{\omega}=g$ together with the stability estimate

$$
\left\|\nabla u_{\omega}\right\|_{0, \omega} \leq \kappa\|g\|_{0, \omega}, \quad \kappa:=\min \left\{\kappa\left(\omega, \gamma_{D}\right), \kappa(\omega, \gamma)\right\}
$$

is provided by Lemma 1.1. Note that $u_{\omega} \in \mathrm{H}_{\gamma}^{1}(\omega)$ can also be regarded as a suitable function if the homogeneous Dirichlet boundary conditions are imposed only on a part $\Gamma_{D}$ of $\Gamma$, so that we can operate with one and the same constant $\kappa(\omega, \gamma)$. Since $u_{\omega}=0$ on $S_{r_{2}}$,

$$
v_{\omega}:= \begin{cases}u_{\omega} & \text { in } \omega, \\ 0 & \text { in } \mathbb{R}^{d} \backslash \bar{B}_{r_{2}}\end{cases}
$$

meets our needs. Then $v_{h}=\eta v+v_{\omega} \in \mathrm{H}_{-1, \Gamma_{D}}^{1}(\Omega)$ and

$$
\operatorname{div} v_{h}=\eta h+\nabla \eta \cdot v+g=h \quad \text { in } \Omega .
$$

It remains to estimate $\left\|\nabla v_{h}\right\|_{0, \Omega}$. By the properties of $\eta$ we have

$$
\|\nabla \eta \otimes v\|_{0, \mathbb{R}^{d}}, \quad\|\nabla \eta \cdot v\|_{0, \mathbb{R}^{d}} \leq \alpha\|v\|_{0, B_{r_{2}} \backslash \bar{B}_{r_{1}}},
$$

where

$$
\alpha:=\alpha\left(r_{1}, r_{2}, \eta\right):=\max _{x \in \mathbb{R}^{d}}|\nabla \eta(x)|=\max _{x \in \bar{B}_{r_{2}} \backslash B_{r_{1}}}|\nabla \eta(x)| .
$$

Since

$$
\|g\|_{0, \omega} \leq\|(1-\eta) h\|_{0, \omega}+\|\nabla \eta \cdot v\|_{0, \omega}
$$

by (30) we find

$$
\begin{aligned}
\left\|\nabla v_{\omega}\right\|_{0, \Omega} & =\left\|\nabla u_{\omega}\right\|_{0, \omega} \\
& \leq \kappa\|g\|_{0, \omega} \leq \kappa\left(\|(1-\eta) h\|_{0, \omega}+\alpha\|v\|_{0, B_{r_{2}} \backslash \bar{B}_{r_{1}}}\right) \\
& \leq \kappa\left(\|h\|_{0, \Omega}+\alpha\|v\|_{0, B_{r_{2}} \backslash \bar{B}_{r_{1}}}\right), \\
\|\nabla(\eta v)\|_{0, \Omega} & \leq\|h\|_{0, \Omega}+\alpha\|v\|_{0, B_{r_{2} \backslash} \backslash \bar{B}_{r_{1}}} .
\end{aligned}
$$

By (26), the second terms on the right-hand sides can be estimated as follows:

$$
\|v\|_{0, B_{r_{2}} \backslash \bar{B}_{r_{1}}} \leq \rho\left(r_{2}\right)\|v\|_{-1, \mathbb{R}^{d}} \leq c_{d} \rho\left(r_{2}\right)\|\nabla v\|_{0, \mathbb{R}^{d}}=c_{d} \rho\left(r_{2}\right)\|h\|_{0, \Omega} .
$$

Finally, by (27), (32), and (33), we conclude that

$$
\left\|\nabla v_{h}\right\|_{0, \Omega} \leq(1+\kappa)\left(\|h\|_{0, \Omega}+c_{d} \alpha \rho\left(r_{2}\right)\|h\|_{0, \Omega}\right)=\kappa_{\oplus}\left(\Omega, \Gamma_{D}\right)\|h\|_{0, \Omega},
$$

finishing the proof. 
3.1. Estimates of the distance to the set $S_{-1, \Gamma_{D}}(\Omega)$. As in the case of bounded domains, the stability Lemma 2.3 implies estimates of the quantity

$$
\operatorname{dist}\left(u, \mathrm{~S}_{-1, \Gamma_{D}}(\Omega)\right)=\inf _{\varphi \in \mathrm{S}_{-1, \Gamma_{D}}(\Omega)}\|\nabla(u-\varphi)\|_{0, \Omega},
$$

which is a measure of the distance between $u \in \mathrm{H}_{-1, \Gamma_{D}}^{1}(\Omega)$ and the corresponding set of divergence free vector fields.

Lemma 3.4. For any $u \in \mathrm{H}_{-1, \Gamma_{D}}^{1}(\Omega)$ there exists a solenoidal $u_{0} \in \mathrm{S}_{-1, \Gamma_{D}}(\Omega)$ such that

$$
\operatorname{dist}\left(u, \mathrm{~S}_{-1, \Gamma_{D}}(\Omega)\right) \leq\left\|\nabla\left(u-u_{0}\right)\right\|_{0, \Omega} \leq \kappa\left(\Omega, \Gamma_{D}\right)\|\operatorname{div} u\|_{0, \Omega} .
$$

Proof. Let $h=\operatorname{div} u \in \mathrm{L}^{2}(\Omega)$. By Lemma 2.3 there exists $u_{h} \in \mathrm{H}_{-1, \Gamma_{D}}^{1}(\Omega)$ such that $\operatorname{div} u_{h}=h$ and

$$
\left\|\nabla u_{h}\right\|_{0, \Omega} \leq \kappa\left(\Omega, \Gamma_{D}\right)\|h\|_{0, \Omega} .
$$

Then $u_{0}:=u-u_{h} \in \mathrm{S}_{-1, \Gamma_{D}}(\Omega)$ and we have

$$
\left\|\nabla\left(u-u_{0}\right)\right\|_{0, \Omega}=\left\|\nabla u_{h}\right\|_{0, \Omega} \leq \kappa\left(\Omega, \Gamma_{D}\right)\|\operatorname{div} u\|_{0, \Omega} .
$$

It is easy to extend this result to the case where $v$ satisfies inhomogeneous Dirichlet boundary conditions on a part of the boundary.

Corollary 3.5. For any $u \in \mathrm{H}_{-1}^{1}(\Omega)$ there exists a solenoidal $u_{0} \in \mathrm{S}_{-1}(\Omega)$ such that $u-u_{0} \in \mathrm{H}_{-1, \Gamma_{D}}^{1}(\Omega)$, i.e., $\left.u_{0}\right|_{\Gamma_{D}}=\left.u\right|_{\Gamma_{D}}$, and

$$
\left\|\nabla\left(u-u_{0}\right)\right\|_{0, \Omega} \leq \kappa\left(\Omega, \Gamma_{D}\right)\|\operatorname{div} u\|_{0, \Omega}
$$

The last assertion shows the existence of a continuous lifting operator that lifts the boundary datum $\left.u\right|_{\Gamma_{D}}$ to the domain $\Omega$ with a solenoidal representative.

\section{§4. A posteriori ESTIMATES}

A posteriori estimates of functional type for various elliptic problems in exterior domains were derived in [27]. In this section, we derive estimates of the difference between the exact solution of the exterior Stokes problem (17)-(21), respectively, (24)-(25) (presented by the fields of velocity $u$, pressure $p$, and stress $\sigma$ ) and the respective approximations $\widetilde{u}, \widetilde{p}$, and $\widetilde{\sigma}$.

First, we introduce two more weighted spaces for tensor valued functions:

$$
\mathbb{D}(\Omega):=\left\{\tau \in \mathrm{L}^{2}(\Omega): \operatorname{div} \tau \in \mathrm{L}_{1}^{2}(\Omega)\right\},
$$

where div is the divergence operator for tensor fields acting as the usual row-wise divergence. $\mathbb{D}(\Omega)$ is a Hilbert space with norm defined by

$$
\|\tau\|_{\mathrm{D}}^{2}:=\|\tau\|_{0, \Omega}^{2}+\|\operatorname{div} \tau\|_{1, \Omega}^{2} .
$$

By $\mathbb{D}_{\Gamma_{N}}(\Omega)$ we denote the closure of $\mathrm{C}_{\Gamma_{N}}^{\infty}(\Omega)$-tensor fields in the norm of $\mathbb{D}(\Omega)$. Then for all $\varphi \in \mathrm{H}_{-1, \Gamma_{D}}^{1}(\Omega)$ and all $\tau \in \mathbb{D}_{\Gamma_{N}}(\Omega)$, we observe that

$$
\langle\tau, \nabla \varphi\rangle_{0, \Omega}+\langle\operatorname{div} \tau, \varphi\rangle_{0, \Omega}=0 .
$$

Notice that

$$
\langle\operatorname{div} \tau, \varphi\rangle_{0, \Omega}=\left\langle\rho \operatorname{div} \tau, \rho^{-1} \varphi\right\rangle_{0, \Omega},
$$

so that the second term in the above relation is well defined. 
4.1. Estimates for the velocity. From now on,

$$
\widetilde{u} \in \mathrm{L}_{-1}^{2}(\Omega) \text { and } \tilde{p} \in \mathrm{L}^{2}(\Omega)
$$

are viewed as approximations of our exact solutions

$$
u=u_{D}+\widehat{u} \in u_{D}+\mathrm{S}_{-1, \Gamma_{D}}(\Omega) \subset \mathrm{S}_{-1}(\Omega) \text { and } p \in \mathrm{L}^{2}(\Omega)
$$

to our exterior Stokes problem (24)-(25), respectively. By

$$
\tilde{\sigma} \in \mathrm{L}^{2}(\Omega)
$$

we denote an approximation of the tensor field

$$
\sigma=\nu \nabla u-p \mathbb{I},
$$

which can be constructed by a certain reconstruction of $\widetilde{u}$ and $\widetilde{p}$ or computed independently.

First, we consider the simplest case where

$$
\widetilde{u} \in u_{D}+\mathrm{S}_{-1, \Gamma_{D}}(\Omega) \subset \mathrm{S}_{-1}(\Omega) .
$$

Then by (24) for all solenoidal $\varphi \in \mathrm{S}_{-1, \Gamma_{D}}(\Omega)$ we have

$$
\langle\nu \nabla(u-\widetilde{u}), \nabla \varphi\rangle_{0, \Omega}=\langle f, \varphi\rangle_{0, \Omega}-\langle\nu \nabla \widetilde{u}, \nabla \varphi\rangle_{0, \Omega} .
$$

Let $\tau \in \mathbb{D}_{\Gamma_{N}}(\Omega)$ and $q \in \mathrm{L}^{2}(\Omega)$. Using Lemma 2.1, identity (34), and the relation

$$
\langle q \mathbb{I}, \nabla \varphi\rangle_{0, \Omega}=0
$$

(which is true for all $\varphi \in \mathrm{S}_{-1}(\Omega)$ because $\mathbb{I}: \nabla \varphi=\operatorname{div} \varphi$ ), we find

$$
\begin{aligned}
& \langle\nu \nabla(u-\widetilde{u}), \nabla \varphi\rangle_{0, \Omega}=\langle\operatorname{div} \tau+f, \varphi\rangle_{0, \Omega}+\langle\tau+q \mathbb{I}-\nu \nabla \widetilde{u}, \nabla \varphi\rangle_{0, \Omega} \\
& \leq\|\operatorname{div} \tau+f\|_{1, \Omega}\|\varphi\|_{-1, \Omega}+\left\|\nu^{-1 / 2}(\tau+q \mathbb{I}-\nu \nabla \widetilde{u})\right\|_{0, \Omega}\left\|\nu^{1 / 2} \nabla \varphi\right\|_{0, \Omega} \\
& \leq\left(\nu_{\ominus}^{-1 / 2} c_{\mathrm{FP}}\left(\Omega, \Gamma_{D}\right)\|\operatorname{div} \tau+f\|_{1, \Omega}+\left\|\nu^{-1 / 2}(\tau+q \mathbb{I}-\nu \nabla \widetilde{u})\right\|_{0, \Omega}\right)\left\|\nu^{1 / 2} \nabla \varphi\right\|_{0, \Omega} .
\end{aligned}
$$

Choosing $\varphi=u-\widetilde{u} \in \mathrm{S}_{-1, \Gamma_{D}}(\Omega)$, we arrive at the following estimate.

Theorem 4.1. Let $\widetilde{u} \in u_{D}+\mathrm{S}_{-1, \Gamma_{D}}(\Omega)$. Then for all $\tau \in \mathbb{D}_{\Gamma_{N}}(\Omega)$ and all $q \in \mathrm{L}^{2}(\Omega)$ we have

$$
\left\|\nu^{1 / 2} \nabla(u-\widetilde{u})\right\|_{0, \Omega} \leq \nu_{\ominus}^{-1 / 2} c_{\mathrm{FP}}\left(\Omega, \Gamma_{D}\right)\|\operatorname{div} \tau+f\|_{1, \Omega}+\left\|\nu^{-1 / 2}(\tau+q \mathbb{I}-\nu \nabla \widetilde{u})\right\|_{0, \Omega} .
$$

The upper bound coincides with the norm of the error on the left-hand side if $\tau=\sigma$ (i.e., $\tau$ coincides with the exact stress tensor) and $q=p$ (i.e., $q$ represents the exact pressure $p$ ). In other words, we have the principal error identity

$$
\begin{aligned}
& \left\|\nu^{1 / 2} \nabla(u-\widetilde{u})\right\|_{0, \Omega} \\
& \quad=\min _{\substack{\tau \in \mathbb{D}_{\Gamma_{N}}(\Omega) \\
q \in \mathrm{L}^{2}(\Omega)}}\left(\nu_{\ominus}^{-1 / 2} c_{\mathrm{FP}}\left(\Omega, \Gamma_{D}\right)\|\operatorname{div} \tau+f\|_{1, \Omega}+\left\|\nu^{-1 / 2}(\tau+q \mathbb{I}-\nu \nabla \widetilde{u})\right\|_{0, \Omega}\right)
\end{aligned}
$$

and the minimum is attained at $\tau=\sigma$ and $q=p$. Similar identities are fulfilled for many other linear elliptic problems. However, Theorem 4.1 has a drawback: the estimate is valid only for those approximate vector fields $\widetilde{u}$ that exactly satisfy the solenoidal condition and the boundary condition. In practice, the solenoidal requirement is difficult to fulfil and approximations arising in 'real life' computations often satisfy the solenoidal condition only approximately. Therefore, our next goal is to extend the estimate to a wider class of nonsolenoidal vector fields. Below we extend the last estimate to a wider class including nonsolenoidal vector functions and assume only that

$$
\widetilde{u} \in u_{D}+\mathrm{H}_{-1, \Gamma_{D}}^{1}(\Omega) \subset \mathrm{H}_{-1}^{1}(\Omega),
$$


i.e., $\widetilde{u}$ is possibly nonsolenoidal but satisfies the boundary condition exactly. Corollary 3.5 guarantees the existence of

$$
u_{0} \in \mathrm{S}_{-1}(\Omega)
$$

such that

$$
u_{0}-\widetilde{u} \in \mathrm{H}_{-1, \Gamma_{D}}^{1}(\Omega)
$$

and

$$
\left\|\nabla\left(u_{0}-\widetilde{u}\right)\right\|_{0, \Omega} \leq \kappa\left(\Omega, \Gamma_{D}\right)\|\operatorname{div} \widetilde{u}\|_{0, \Omega} .
$$

Hence $u_{0}=\widetilde{u}+u_{0}-\widetilde{u} \in u_{D}+\mathrm{H}_{-1, \Gamma_{D}}^{1}(\Omega)$, i.e., $u_{0} \in u_{D}+\mathrm{S}_{-1, \Gamma_{D}}(\Omega)$, and by Theorem 4.1 we have

$$
\begin{aligned}
\left\|\nu^{1 / 2} \nabla(u-\widetilde{u})\right\|_{0, \Omega} \leq & \left\|\nu^{1 / 2} \nabla\left(u-u_{0}\right)\right\|_{0, \Omega}+\left\|\nu^{1 / 2} \nabla\left(u_{0}-\widetilde{u}\right)\right\|_{0, \Omega} \\
\leq & \nu_{\ominus}^{-1 / 2} c_{\mathrm{FP}}\left(\Omega, \Gamma_{D}\right)\|\operatorname{div} \tau+f\|_{1, \Omega} \\
& +\left\|\nu^{-1 / 2}\left(\tau+q \mathbb{I}-\nu \nabla u_{0}\right)\right\|_{0, \Omega}+\left\|\nu^{1 / 2} \nabla\left(u_{0}-\widetilde{u}\right)\right\|_{0, \Omega} \\
\leq & \nu_{\ominus}^{-1 / 2} c_{\mathrm{FP}}\left(\Omega, \Gamma_{D}\right)\|\operatorname{div} \tau+f\|_{1, \Omega} \\
& +\left\|\nu^{-1 / 2}(\tau+q \mathbb{I}-\nu \nabla \widetilde{u})\right\|_{0, \Omega}+2 \nu_{\oplus}^{1 / 2}\left\|\nabla\left(u_{0}-\widetilde{u}\right)\right\|_{0, \Omega} .
\end{aligned}
$$

In view of (36), we obtain the following result.

Theorem 4.2. Let $\widetilde{u} \in u_{D}+\mathrm{H}_{-1, \Gamma_{D}}^{1}(\Omega)$. Then for all $\tau \in \mathbb{D}_{\Gamma_{N}}(\Omega)$ and all $q \in \mathrm{L}^{2}(\Omega)$ we have

$$
\begin{aligned}
\left\|\nu^{1 / 2} \nabla(u-\widetilde{u})\right\|_{0, \Omega} & \leq \nu_{\ominus}^{-1 / 2} c_{\mathrm{FP}}\left(\Omega, \Gamma_{D}\right)\|\operatorname{div} \tau+f\|_{1, \Omega} \\
& +\left\|\nu^{-1 / 2}(\tau+q \mathbb{I}-\nu \nabla \widetilde{u})\right\|_{0, \Omega}+2 \nu_{\oplus}^{1 / 2} \kappa\left(\Omega, \Gamma_{D}\right)\|\operatorname{div} \widetilde{u}\|_{0, \Omega} .
\end{aligned}
$$

If the approximation $\widetilde{u}$ is solenoidal, we recover Theorem 4.1 and, again, the upper bound coincides with the norm of the error on the left-hand side if $\tau=\sigma, q=p$. If the approximation $\widetilde{u}$ is solenoidal only in, e.g., $\mathbb{R}^{d} \backslash \bar{B}_{r_{2}}$, then we trivially get an estimate by Theorem 4.2, replacing the term $\|\operatorname{div} \widetilde{u}\|_{0, \Omega}$ by $\|\operatorname{div} \widetilde{u}\|_{0, \omega}$. But with a moderate additional assumption on the decay of the approximation we can even do better in this case, replacing the constant $\kappa\left(\Omega, \Gamma_{D}\right)$ by a stability constant $\kappa\left(\omega, \gamma_{D}\right)$ of the bounded domain $\omega$.

For this let

$$
\widetilde{u}=u_{D}+w \in u_{D}+\mathrm{H}_{-1, \Gamma_{D}}^{1}(\Omega)
$$

with $\operatorname{div} \widetilde{u}=\operatorname{div} w=0$ in $\mathbb{R}^{d} \backslash \bar{B}_{r_{2}}$ and if $\gamma_{D}=\gamma$ (i.e., $\Gamma_{D}=\Gamma$ ), then $\widetilde{u}$ satisfies $\operatorname{div} \widetilde{u} \in L_{\perp}^{2}(\omega)$. To meet the last condition, we additionally assume for the case of $\Gamma_{D}=\Gamma$ that

$$
|w| \leq c r^{-m}, \quad m>d-1
$$

as $r \rightarrow \infty$ with some $c>0$ independent of $r$ (notice that $r^{-m} \in \mathrm{L}_{-1}^{2}\left(\mathbb{R}^{d} \backslash \bar{B}_{1}\right.$ ) if $m>d / 2-1)$. Indeed, it is easy to see that

$$
\left|\int_{\omega} \operatorname{div} \widetilde{u}\right|=\left|\int_{\omega} \operatorname{div} w\right|=\left|\int_{S_{r}} n \cdot w\right| \leq c r^{d-1-m} \stackrel{r \rightarrow \infty}{\longrightarrow} 0
$$

for $r>r_{2}$. Now we consider the Ansatz

$$
u_{0}:=\widetilde{u}+ \begin{cases}u_{\omega} & \text { in } \omega, \\ 0 & \text { in } \mathbb{R}^{d} \backslash \bar{B}_{r_{2}},\end{cases}
$$

where $u_{\omega} \in \mathrm{H}_{\gamma_{D}}^{1}(\omega)$. Utilising Lemma 1.1, we find $u_{\omega} \in \mathrm{H}_{\gamma_{D}}^{1}(\omega)$ such that

$$
\operatorname{div} u_{\omega}=-\operatorname{div} \widetilde{u} \text { in } \omega
$$


together with the stability estimate

$$
\left\|\nabla u_{\omega}\right\|_{0, \omega} \leq \kappa\left(\omega, \gamma_{D}\right)\|\operatorname{div} \widetilde{u}\|_{0, \omega} .
$$

The function $u_{0}$ so constructed satisfies the boundary condition on $\Gamma_{D}$ and it is a solenoidal field, i.e.,

$$
u_{0} \in u_{D}+\mathrm{S}_{-1, \Gamma_{D}}(\Omega) .
$$

Using $u_{0}$ as an admissible vector field in (37) yields the estimate

$$
\begin{aligned}
\left\|\nu^{1 / 2} \nabla(u-\widetilde{u})\right\|_{0, \Omega} \leq & \nu_{\ominus}^{-1 / 2} c_{\mathrm{FP}}\left(\Omega, \Gamma_{D}\right)\|\operatorname{div} \tau+f\|_{1, \Omega} \\
& +\left\|\nu^{-1 / 2}(\tau+q \mathbb{I}-\nu \nabla \widetilde{u})\right\|_{0, \Omega}+2 \nu_{\oplus}^{1 / 2} \underbrace{\left\|\nabla\left(u_{0}-\widetilde{u}\right)\right\|_{0, \Omega}}_{=\left\|\nabla u_{\omega}\right\|_{0, \omega}} .
\end{aligned}
$$

Hence we have the following improved estimate for the case of a partially solenoidal approximation.

Corollary 4.3. Let $\widetilde{u} \in u_{D}+\mathrm{H}_{-1, \Gamma_{D}}^{1}(\Omega)$ and let

$$
\operatorname{div} \widetilde{u}=0 \quad \text { in } \mathbb{R}^{d} \backslash \bar{B}_{r_{2}} .
$$

If $\Gamma_{D}=\Gamma$, then we additionally impose condition (38). Then for all $\tau \in \mathbb{D}_{\Gamma_{N}}(\Omega)$ and all $q \in \mathrm{L}^{2}(\Omega)$ we have

$$
\begin{aligned}
\left\|\nu^{1 / 2} \nabla(u-\widetilde{u})\right\|_{0, \Omega} & \leq \nu_{\ominus}^{-1 / 2} c_{\mathrm{FP}}\left(\Omega, \Gamma_{D}\right)\|\operatorname{div} \tau+f\|_{1, \Omega} \\
& +\left\|\nu^{-1 / 2}(\tau+q \mathbb{I}-\nu \nabla \widetilde{u})\right\|_{0, \Omega}+2 \nu_{\oplus}^{1 / 2} \kappa\left(\omega, \gamma_{D}\right)\|\operatorname{div} \widetilde{u}\|_{0, \omega} .
\end{aligned}
$$

Here the last term on the right-hand side is a penalty for a possible violation of the solenoidal condition in $\omega$.

4.2. Estimates for the pressure. By Lemma 2.3 there exists a vector field $\psi \in$ $\mathrm{H}_{-1, \Gamma_{D}}^{1}(\Omega)$ such that

$$
\operatorname{div} \psi=p-\widetilde{p} \quad \text { and } \quad\|\nabla \psi\|_{0, \Omega} \leq \kappa\left(\Omega, \Gamma_{D}\right)\|p-\widetilde{p}\|_{0, \Omega} .
$$

For all $\widetilde{u} \in u_{D}+\mathrm{H}_{-1, \Gamma_{D}}^{1}(\Omega)$ and all $\tau \in \mathbb{D}_{\Gamma_{N}}(\Omega)$ we have

$$
\begin{aligned}
\|p-\widetilde{p}\|_{0, \Omega}^{2}= & \langle p-\widetilde{p}, \operatorname{div} \psi\rangle_{0, \Omega} \\
= & \langle\nu \nabla(u-\widetilde{u}), \nabla \psi\rangle_{0, \Omega}-\langle\operatorname{div} \tau+f, \psi\rangle_{0, \Omega}+\langle\nu \nabla \widetilde{u}-\widetilde{p} \mathbb{I}-\tau, \nabla \psi\rangle_{0, \Omega} \\
\leq & \left(\|\nu \nabla(u-\widetilde{u})\|_{0, \Omega}+c_{\mathrm{FP}}\left(\Omega, \Gamma_{D}\right)\|\operatorname{div} \tau+f\|_{1, \Omega}\right. \\
& \left.+\|\nu \nabla \widetilde{u}-\widetilde{p} \mathbb{I}-\tau\|_{0, \Omega}\right)\|\nabla \psi\|_{0, \Omega},
\end{aligned}
$$

where we have used Lemma 2.1 for $\psi$ and the relation $\operatorname{div} \psi=\mathbb{I}: \nabla \psi$. By (39) we obtain

$$
\begin{aligned}
\|p-\widetilde{p}\|_{0, \Omega} & \leq \kappa\left(\Omega, \Gamma_{D}\right)\left(\nu_{\oplus}^{1 / 2}\left\|\nu^{1 / 2} \nabla(u-\widetilde{u})\right\|_{0, \Omega}\right. \\
& \left.+c_{\mathrm{FP}}\left(\Omega, \Gamma_{D}\right)\|\operatorname{div} \tau+f\|_{1, \Omega}+\nu_{\oplus}^{1 / 2}\left\|\nu^{-1 / 2}(\tau+\widetilde{p} \mathbb{I}-\nu \nabla \widetilde{u})\right\|_{0, \Omega}\right) .
\end{aligned}
$$

In order to estimate the first term on the right-hand side, we use Theorem 4.2 with $q=\widetilde{p}$ and arrive at the desired estimate for the pressure field.

Theorem 4.4. Let $\widetilde{p} \in \mathrm{L}^{2}(\Omega)$. Then for all $\tau \in \mathbb{D}_{\Gamma_{N}}(\Omega)$ and all

$$
\widetilde{u} \in u_{D}+\mathrm{H}_{-1, \Gamma_{D}}^{1}(\Omega)
$$


we have

$$
\begin{aligned}
\|p-\widetilde{p}\|_{0, \Omega} \leq & \kappa\left(\Omega, \Gamma_{D}\right)\left(\left(\nu_{\ominus}^{-1 / 2} \nu_{\oplus}^{1 / 2}+1\right) c_{\mathrm{FP}}\left(\Omega, \Gamma_{D}\right)\|\operatorname{div} \tau+f\|_{1, \Omega}\right. \\
& \left.+2 \nu_{\oplus}^{1 / 2}\left\|\nu^{-1 / 2}(\tau+\widetilde{p} \mathbb{I}-\nu \nabla \widetilde{u})\right\|_{0, \Omega}+2 \nu_{\oplus} \kappa\left(\Omega, \Gamma_{D}\right)\|\operatorname{div} \widetilde{u}\|_{0, \Omega}\right) .
\end{aligned}
$$

Remark 4.5. The upper bound (40) consists of the same terms as the upper bound of Theorem 4.2 and vanishes if $\widetilde{u}=u, \tau=\sigma, \widetilde{p}=p$. However, in this case, the estimate stronger depends on the stability constant $\kappa\left(\Omega, \Gamma_{D}\right)$. A similar effect occurs in the estimates related to bounded domains (see $[35,36]$ ).

4.3. Estimates for nonconforming approximations. The term nonconforming is usually applied to approximations that belong to a functional class wider than the natural energy class of the problem in question. For example, nonconformity of approximations may arise due to violation of continuity conditions or main boundary conditions. Nowadays such type approximations are widely used in computational practice (e.g., mortar, finite volume, and discontinuous Galerkin approximations) because they offer more freedom for various mesh adaptive procedures. Application of functional type $a$ posteriori estimates to nonconforming approximations of elliptic problems was studied earlier in $[4,15,37,45]$. In this section, we briefly discuss this question in the context of the exterior Stokes problem.

Let us now assume that we have a nonconforming approximation

$$
\widetilde{\Upsilon} \in \mathrm{L}^{2}(\Omega)
$$

of the exact strain tensor field

$$
\Upsilon:=\nabla u, \quad u=u_{D}+\widehat{u} \in u_{D}+\mathrm{S}_{-1, \Gamma_{D}}(\Omega) \subset \mathrm{S}_{-1}(\Omega) .
$$

For example, $\widetilde{\Upsilon}$ as a "broken gradient" tensor field, the output of some discontinuous Galerkin method. By the triangle inequality, we estimate the difference between these tensor fields using a certain conforming approximation $\widetilde{u} \in u_{D}+\mathrm{H}_{-1, \Gamma_{D}}^{1}(\Omega)$ and obtain

$$
\left\|\nu^{1 / 2}(\Upsilon-\widetilde{\Upsilon})\right\|_{0, \Omega} \leq\left\|\nu^{1 / 2} \nabla(u-\widetilde{u})\right\|_{0, \Omega}+\left\|\nu^{1 / 2}(\nabla \widetilde{u}-\widetilde{\Upsilon})\right\|_{0, \Omega}
$$

Theorem 4.2 implies the estimate

$$
\begin{aligned}
\left\|\nu^{1 / 2}(\Upsilon-\widetilde{\Upsilon})\right\|_{0, \Omega} & \leq \nu_{\ominus}^{-1 / 2} c_{\mathrm{FP}}\left(\Omega, \Gamma_{D}\right)\|\operatorname{div} \tau+f\|_{1, \Omega}+\left\|\nu^{-1 / 2}(\tau+q \mathbb{I}-\nu \nabla \widetilde{u})\right\|_{0, \Omega} \\
& +2 \nu_{\oplus}^{1 / 2} \kappa\left(\Omega, \Gamma_{D}\right)\|\operatorname{div} \widetilde{u}\|_{0, \Omega}+\left\|\nu^{1 / 2}(\nabla \widetilde{u}-\widetilde{\Upsilon})\right\|_{0, \Omega} .
\end{aligned}
$$

Using the triangle inequality and Theorem 4.4, we obtain a posteriori error estimates for nonconforming approximations.

Theorem 4.6. Let $\widetilde{\Upsilon} \in \mathrm{L}^{2}(\Omega)$ and let $\widetilde{p} \in \mathrm{L}^{2}(\Omega)$. Then for all $\tau \in \mathbb{D}_{\Gamma_{N}}(\Omega), q \in \mathrm{L}^{2}(\Omega)$, and $\widetilde{u} \in u_{D}+\mathrm{H}_{-1, \Gamma_{D}}^{1}(\Omega)$ we have

$$
\begin{gathered}
\left\|\nu^{1 / 2}(\Upsilon-\widetilde{\Upsilon})\right\|_{0, \Omega} \leq \nu_{\ominus}^{-1 / 2} c_{\mathrm{FP}}\left(\Omega, \Gamma_{D}\right)\|\operatorname{div} \tau+f\|_{1, \Omega}+\left\|\nu^{-1 / 2}(\tau+q \mathbb{I}-\nu \widetilde{\Upsilon})\right\|_{0, \Omega} \\
+2 \nu_{\oplus}^{1 / 2} \kappa\left(\Omega, \Gamma_{D}\right)\|\operatorname{div} \widetilde{u}\|_{0, \Omega}+2\left\|\nu^{1 / 2}(\nabla \widetilde{u}-\widetilde{\Upsilon})\right\|_{0, \Omega}
\end{gathered}
$$

and

$$
\begin{aligned}
\|p-\widetilde{p}\|_{0, \Omega} \leq & \kappa\left(\Omega, \Gamma_{D}\right)\left(\left(\nu_{\ominus}^{-1 / 2} \nu_{\oplus}^{1 / 2}+1\right) c_{\mathrm{FP}}\left(\Omega, \Gamma_{D}\right)\|\operatorname{div} \tau+f\|_{1, \Omega}\right. \\
+ & 2 \nu_{\oplus}^{1 / 2}\left\|\nu^{-1 / 2}(\tau+\widetilde{p} \mathbb{I}-\nu \widetilde{\Upsilon})\right\|_{0, \Omega}+2 \nu_{\oplus} \kappa\left(\Omega, \Gamma_{D}\right)\|\operatorname{div} \widetilde{u}\|_{0, \Omega} \\
& \left.+2 \nu_{\oplus}^{1 / 2}\left\|\nu^{1 / 2}(\nabla \widetilde{u}-\widetilde{\Upsilon})\right\|_{0, \Omega}\right) .
\end{aligned}
$$


It is easy to see that in the case where $\widetilde{\Upsilon}=\nabla \widetilde{u}$ is generated by the conforming approximation $\widetilde{u} \in u_{D}+\mathrm{H}_{-1, \Gamma_{D}}^{1}(\Omega)$, the last term vanishes and we recover Theorem 4.2 and Theorem 4.4.

4.4. Estimates for the stress field. Error estimates for the stress tensor field follow directly from the estimates derived above for the velocity vector field and the pressure function. Indeed, let $\widetilde{\sigma} \in \mathrm{L}^{2}(\Omega)$ be an approximation of the exact stress tensor

$$
\sigma=\nu \nabla u-p \mathbb{I}=\nu \Upsilon-p \mathbb{I} .
$$

Moreover, let $\widetilde{\Upsilon} \in \mathrm{L}^{2}(\Omega)$ and $\widetilde{p} \in \mathrm{L}^{2}(\Omega)$. Then, the respective error estimate follows from the triangle inequality

$$
\|\widetilde{\sigma}-\sigma\|_{0, \Omega} \leq\|\widetilde{\sigma}-\nu \widetilde{\Upsilon}+\widetilde{p} \mathbb{I}\|_{0, \Omega}+\nu_{\oplus}^{1 / 2}\left\|\nu^{1 / 2}(\Upsilon-\widetilde{\Upsilon})\right\|_{0, \Omega}+d^{1 / 2}\|p-\widetilde{p}\|_{0, \Omega}
$$

In particular, we can set $\widetilde{\Upsilon}=\nabla \widetilde{u}$, where $\widetilde{u} \in u_{D}+\mathrm{H}_{-1, \Gamma_{D}}^{1}(\Omega)$. The first term on the righthand side involves only known tensor fields and the second and third ones are estimated by, e.g., Theorem 4.2, Theorem 4.4, and Theorem 4.6.

\subsection{Lower bounds of the error. Let}

$$
\widetilde{u} \in u_{D}+\mathrm{H}_{-1, \Gamma_{D}}^{1}(\Omega)
$$

i.e., $u-\widetilde{u} \in \mathrm{H}_{-1, \Gamma_{D}}^{1}(\Omega)$. Obviously (since the subsequent max-property is true for any Hilbert $^{3}$ space), by (24) we have

$$
\begin{aligned}
\| \nu^{1 / 2} & \nabla(u-\widetilde{u}) \|_{0, \Omega}^{2}=\max _{\varphi \in \mathrm{H}_{-1, \Gamma_{D}}^{1}(\Omega)}\left(2\langle\nu \nabla(u-\widetilde{u}), \nabla \varphi\rangle_{0, \Omega}-\left\|\nu^{1 / 2} \nabla \varphi\right\|_{0, \Omega}^{2}\right) \\
& \geq 2\langle\nu \nabla u, \nabla \varphi\rangle_{0, \Omega}-2\langle\nu \nabla \widetilde{u}, \nabla \varphi\rangle_{0, \Omega}-\left\|\nu^{1 / 2} \nabla \varphi\right\|_{0, \Omega}^{2} \\
& =2\langle f, \varphi\rangle_{0, \Omega}+2\langle q, \operatorname{div} \varphi\rangle_{0, \Omega}-\langle\nu \nabla(2 \widetilde{u}+\varphi), \nabla \varphi\rangle_{0, \Omega}+2\langle p-q, \operatorname{div} \varphi\rangle_{0, \Omega}
\end{aligned}
$$

and the maximum is attained at $\varphi=u-\widetilde{u} \in \mathrm{H}_{-1, \Gamma_{D}}^{1}(\Omega)$. The last term can be simply (but rather coarsly) estimated by Theorem $4.4(\widetilde{p}=q)$, what yields the estimate presented below.

Theorem 4.7. For all $\tilde{u}, v \in u_{D}+\mathrm{H}_{-1, \Gamma_{D}}^{1}(\Omega)$ and all $\varphi \in \mathrm{H}_{-1, \Gamma_{D}}^{1}(\Omega), \tau \in \mathbb{D}_{\Gamma_{N}}(\Omega)$, and $q \in \mathrm{L}^{2}(\Omega)$ we have

$$
\begin{aligned}
\left\|\nu^{1 / 2} \nabla(u-\widetilde{u})\right\|_{0, \Omega}^{2} \geq & 2\langle f, \varphi\rangle_{0, \Omega}+2\langle q, \operatorname{div} \varphi\rangle_{0, \Omega}-\langle\nu \nabla(2 \widetilde{u}+\varphi), \nabla \varphi\rangle_{0, \Omega} \\
& -2 \kappa\left(\Omega, \Gamma_{D}\right)\|\operatorname{div} \varphi\|_{0, \Omega}\left(\left(\nu_{\ominus}^{-1 / 2} \nu_{\oplus}^{1 / 2}+1\right) c_{\mathrm{FP}}\left(\Omega, \Gamma_{D}\right)\|\operatorname{div} \tau+f\|_{1, \Omega}\right. \\
& \left.+2 \nu_{\oplus}^{1 / 2}\left\|\nu^{-1 / 2}(\tau+q \mathbb{I}-\nu \nabla v)\right\|_{0, \Omega}+2 \nu_{\oplus} \kappa\left(\Omega, \Gamma_{D}\right)\|\operatorname{div} v\|_{0, \Omega}\right) .
\end{aligned}
$$

In particular, $v=\widetilde{u}$ is possible. If $\varphi \in \mathrm{S}_{-1, \Gamma_{D}}(\Omega)$, then the simple lower bound

$$
\left\|\nu^{1 / 2} \nabla(u-\widetilde{u})\right\|_{0, \Omega}^{2} \geq 2\langle f, \varphi\rangle_{0, \Omega}-\langle\nu \nabla(2 \widetilde{u}+\varphi), \nabla \varphi\rangle_{0, \Omega}
$$

is true. Moreover, equality occurs for $\varphi=u-\widetilde{u}$, provided that the approximation $\widetilde{u}$ is also solenoidal, i.e., $\widetilde{u} \in u_{D}+\mathrm{S}_{-1, \Gamma_{D}}(\Omega)$.

To handle a nonconforming approximation $\widetilde{\Upsilon} \in \mathrm{L}^{2}(\Omega)$, we can simply utilise the triangle inequality and apply Theorem 4.7 .

\footnotetext{
${ }^{3}$ In any Hilbert space $\mathbf{H}$ it is true that $\|x\|^{2}=\max _{y \in \mathrm{H}}\left(2\langle x, y\rangle-\|y\|^{2}\right)$, in our case we can set $\mathbf{H}=L^{2}(\Omega)$.
} 
Theorem 4.8. Let $\widetilde{\Upsilon} \in \mathrm{L}^{2}(\Omega)$. Then for all $\varphi \in \mathrm{H}_{-1, \Gamma_{D}}^{1}(\Omega), \tau \in \mathbb{D}_{\Gamma_{N}}(\Omega), q \in \mathrm{L}^{2}(\Omega)$, and $\widetilde{u}, v \in u_{D}+\mathrm{H}_{-1, \Gamma_{D}}^{1}(\Omega)$, we have

$$
\begin{aligned}
\left\|\nu^{1 / 2}(\Upsilon-\widetilde{\Upsilon})\right\|_{0, \Omega} & \geq\left\|\nu^{1 / 2} \nabla(u-\widetilde{u})\right\|_{0, \Omega}-\left\|\nu^{1 / 2}(\nabla \widetilde{u}-\widetilde{\Upsilon})\right\|_{0, \Omega}, \\
\left\|\nu^{1 / 2} \nabla(u-\widetilde{u})\right\|_{0, \Omega}^{2} & \geq 2\langle f, \varphi\rangle_{0, \Omega}+2\langle q, \operatorname{div} \varphi\rangle_{0, \Omega}-\langle\nu \nabla(2 \widetilde{u}+\varphi), \nabla \varphi\rangle_{0, \Omega} \\
& -2 \kappa\left(\Omega, \Gamma_{D}\right)\|\operatorname{div} \varphi\|_{0, \Omega}\left(\left(\nu_{\ominus}^{-1 / 2} \nu_{\oplus}^{1 / 2}+1\right) c_{\mathrm{FP}}\left(\Omega, \Gamma_{D}\right)\|\operatorname{div} \tau+f\|_{1, \Omega}\right. \\
& \left.+2 \nu_{\oplus}^{1 / 2}\left\|\nu^{-1 / 2}(\tau+q \mathbb{I}-\nu \nabla v)\right\|_{0, \Omega}+2 \nu_{\oplus} \kappa\left(\Omega, \Gamma_{D}\right)\|\operatorname{div} v\|_{0, \Omega}\right) .
\end{aligned}
$$

4.6. Special case: $2 \mathrm{D}$ exterior domains. For a Lipschitz domain $\mathcal{D} \subset \mathbb{R}^{2}$ we introduce somewhat different weighted spaces by using logarithmic weights, namely,

$$
\begin{aligned}
\mathrm{L}_{ \pm 1, \ln }^{2}(\mathcal{D}) & :=\left\{\phi \in \mathrm{L}_{\text {loc }}^{2}(\mathcal{D}):(\rho \ln (e+\rho))^{ \pm 1} \phi \in \mathrm{L}^{2}(\mathcal{D})\right\} \\
\mathrm{H}_{-1, \ln }^{1}(\mathcal{D}) & :=\left\{\phi \in \mathrm{L}_{-1, \ln }^{2}(\mathcal{D}): \nabla \phi \in \mathrm{L}^{2}(\mathcal{D})\right\},
\end{aligned}
$$

where $e$ is the Euler number, see, e.g., $[16,43,9]$. We notice that at infinity $(\rho \ln (e+\rho))^{ \pm 1}$ behaves like $(r \ln r)^{ \pm 1}$. The inner product in $\mathrm{L}_{ \pm 1, \ln }^{2}(\mathcal{D})$ is defined by the relation

$$
\langle\phi, \psi\rangle_{ \pm 1, \ln , \mathcal{D}}:=\left\langle(\rho \ln (e+\rho))^{ \pm 2} \phi, \psi\right\rangle_{0, \mathcal{D}} \text {. }
$$

All other weighted spaces and norms are modified and defined in a similar way.

The sets $\Omega \subset \mathbb{R}^{2}$ and $\omega \subset \mathbb{R}^{2}$ are defined as in $\S 2$, i.e., $\Omega \subset \mathbb{R}^{2}$ is an exterior Lipschitz domain and $\omega$ is a certain truncation of $\Omega$. The situation is now different from the case of $d \geq 3$ because the constants ${ }^{4}$ will be integrable in our weighted spaces, i.e., $1 \in \mathrm{L}_{-1, \ln }^{2}(\Omega)$. Introducing additionally

$$
\mathrm{H}_{-1, \ln , \varnothing}^{1}(\Omega):=\mathrm{H}_{-1, \ln }^{1}(\Omega) \cap \mathbb{R}^{\perp_{-1, \ln , \Omega}}
$$

we have the following Friedrichs/Poincaré estimate for exterior domains.

Lemma 4.9. There exists $c>0$ such that

$$
\|v\|_{-1, \ln , \Omega} \leq c\|\nabla v\|_{0, \Omega}
$$

for all $v \in \mathrm{H}_{-1, \ln , \Gamma_{D}}^{1}(\Omega)$. The best constant $c$ is denoted by $c_{\mathrm{FP}}\left(\Omega, \Gamma_{D}\right)$. In the special case where $B_{e} \subset \mathbb{R}^{d} \backslash \bar{\Omega}$ and $\Gamma_{D}=\Gamma$, we have $c_{\mathrm{FP}}(\Omega, \Gamma) \leq 2$.

This theorem follows from [27, Appendix 4.2, Lemma 4.1, Corollary 4.2, Remark 4.3], see also [43, Lemma 4.1] and $[28,16]$. It should be noted that in this case we need boundary or mean value conditions as in the case of a bounded domain. Now, all results from the sections for $d \geq 3$ follow with obvious modifications. In particular, the stability Lemma 3.2 reads as follows.

Lemma 4.10. There exists $c>0$ such that for any $h \in \mathrm{L}^{2}(\Omega)$ there exists $u_{h} \in$ $\mathrm{H}_{-1, \ln , \Gamma_{D}}^{1}(\Omega)$ with

$$
\operatorname{div} u_{h}=h \quad \text { and } \quad\left\|\nabla u_{h}\right\|_{0, \Omega} \leq c\|h\|_{0, \Omega} .
$$

The best constant is denoted by $\kappa\left(\Omega, \Gamma_{D}\right)$ and satisfies the estimate

$$
\kappa\left(\Omega, \Gamma_{D}\right) \leq \kappa_{\oplus}\left(\Omega, \Gamma_{D}\right):=(1+\kappa)\left(1+c_{\mathrm{P}}\left(\mathbb{R}^{2}\right) \alpha \rho\left(r_{2}\right) \ln \left(e+\rho\left(r_{2}\right)\right)\right)
$$

with

$$
\alpha=\alpha\left(r_{1}, r_{2}, \eta\right)=\max _{x \in \bar{B}_{r_{2}} \backslash B_{r_{1}}}|\nabla \eta(x)| \quad \text { and } \quad \kappa=\min \left\{\kappa\left(\omega, \gamma_{D}\right), \kappa(\omega, \gamma)\right\} .
$$

\footnotetext{
${ }^{4}$ Specifically, $(r \ln r)^{-1} \in \mathrm{L}^{2}\left(B_{\epsilon}\right),(r \ln r)^{-1} \notin \mathrm{L}^{2}\left(B_{1+\epsilon} \backslash \bar{B}_{1-\epsilon}\right),(r \ln r)^{-1} \in \mathrm{L}^{2}\left(\mathbb{R}^{2} \backslash \bar{B}_{1+\epsilon}\right)$ for $0<\epsilon<1$.
} 
If $h$ has compact support in $\bar{\omega}$ and (if $\Gamma_{D}=\Gamma$ ) additionally $\int_{\Omega} h=\int_{\omega} h=0$, then $u_{h}$ can be chosen with a compact support in $\bar{\omega}$ as well, in particular $u_{h} \in \mathrm{H}_{\gamma_{D}}^{1}(\omega) \subset \mathrm{H}_{\Gamma_{D}}^{1}(\Omega)$. In this case, $\kappa\left(\Omega, \Gamma_{D}\right) \leq \kappa\left(\omega, \gamma_{D}\right)$.

Estimates of the distance to the set of solenoidal fields are derived quite similarly.

Corollary 4.11. For any $u \in \mathrm{H}_{-1, \ln , \Gamma_{D}}^{1}(\Omega)$ there exists a solenoidal $u_{0} \in \mathrm{S}_{-1, \ln , \Gamma_{D}}(\Omega)$ such that

$$
\operatorname{dist}\left(u, \mathrm{~S}_{-1, \ln , \Gamma_{D}}(\Omega)\right) \leq\left\|\nabla\left(u-u_{0}\right)\right\|_{0, \Omega} \leq \kappa\left(\Omega, \Gamma_{D}\right)\|\operatorname{div} u\|_{0, \Omega} .
$$

For any $u \in \mathrm{H}_{-1, \ln }^{1}(\Omega)$ there exists a solenoidal $u_{0} \in \mathrm{S}_{-1, \ln }(\Omega)$ such that

$$
u-u_{0} \in \mathrm{H}_{-1, \ln , \Gamma_{D}}^{1}(\Omega),
$$

i.e., $\left.u_{0}\right|_{\Gamma_{D}}=\left.u\right|_{\Gamma_{D}}$, and

$$
\left\|\nabla\left(u-u_{0}\right)\right\|_{0, \Omega} \leq \kappa\left(\Omega, \Gamma_{D}\right)\|\operatorname{div} u\|_{0, \Omega}
$$

Another obvious corollary is the inf-sup lemma for $2 \mathrm{D}$ exterior domains.

Corollary 4.12. We have

$$
\inf _{h \in \mathrm{L}^{2}(\Omega)} \sup _{u \in \mathrm{H}_{-1, \ln , \Gamma_{D}}^{1}(\Omega)} \frac{\langle h, \operatorname{div} u\rangle_{0, \Omega}}{\|h\|_{0, \Omega}\|\nabla u\|_{0, \Omega}} \geq \frac{1}{\kappa\left(\Omega, \Gamma_{D}\right)} .
$$

As in the case of $d \geq 3$, the solvability of the Stokes problem and respective energy estimates follow. Below we recall these results. Let

$$
\mathrm{L}_{1, \ln , \Gamma_{N}}^{2}(\Omega):=\left\{\begin{array}{lll}
\mathrm{L}_{1, \ln }^{2}(\Omega) & \text { if } \Gamma_{D} \neq \varnothing, \\
\mathrm{L}_{1, \ln , \perp}^{2}(\Omega) & \text { if } \Gamma_{D}=\varnothing
\end{array}\right.
$$

and

$$
\mathrm{L}_{1, \ln , \perp}^{2}(\Omega):=\mathrm{L}_{1, \ln }^{2}(\Omega) \cap\left(\mathbb{R}^{2}\right)^{\perp_{0, \Omega}}=\left\{\phi \in \mathrm{L}_{1, \ln }^{2}(\Omega): \int_{\Omega} \phi_{i}=0\right\} .
$$

Corollary 4.13. For $\nu, f \in \mathrm{L}_{1, \ln , \Gamma_{N}}^{2}(\Omega)$, and $u_{D} \in \mathrm{S}_{-1, \ln }(\Omega)$, the $2 D$ Stokes system is uniquely solvable with a solenoidal vector field $u=u_{D}+\widehat{u} \in u_{D}+\mathrm{S}_{-1, \ln , \Gamma_{D}}(\Omega) \subset \mathrm{S}_{-1, \ln }(\Omega)$ and $p \in \mathrm{L}^{2}(\Omega)$. Moreover,

$$
\begin{aligned}
\nu\|\nabla \widehat{u}\|_{0, \Omega} & \leq c_{\mathrm{FP}}\left(\Omega, \Gamma_{D}\right)\|f\|_{1, \ln , \Omega}+\nu\left\|\nabla u_{D}\right\|_{0, \Omega}, \\
\nu\|\nabla u\|_{0, \Omega} & \leq c_{\mathrm{FP}}\left(\Omega, \Gamma_{D}\right)\|f\|_{1, \ln , \Omega}+2 \nu\left\|\nabla u_{D}\right\|_{0, \Omega}, \\
\|p\|_{0, \Omega} & \leq 2 \kappa\left(\Omega, \Gamma_{D}\right)\left(c_{\mathrm{FP}}\left(\Omega, \Gamma_{D}\right)\|f\|_{1, \ln , \Omega}+\nu\left\|\nabla u_{D}\right\|_{0, \Omega}\right) .
\end{aligned}
$$

Now we address the subject of a posteriori error estimation and introduce the sets

$$
\mathbb{D}(\Omega):=\left\{\tau \in \mathrm{L}^{2}(\Omega): \operatorname{div} \tau \in \mathrm{L}_{1, \ln }^{2}(\Omega)\right\}
$$

and $\mathbb{D}_{\Gamma_{N}}(\Omega)$ as the closure of $\mathrm{C}_{\Gamma_{N}}^{\infty}(\Omega)$-tensor fields in the norm of $\mathbb{D}(\Omega)$. For errors encompassed in approximation of the velocity field we have the following results, which repeat (with some modifications) those derived for $d \geq 3$. First, we present an analog of Theorem 4.2 .

Theorem 4.14. Let $\widetilde{u} \in u_{D}+\mathrm{H}_{-1, \ln , \Gamma_{D}}^{1}(\Omega)$. Then for all $\tau \in \mathbb{D}_{\Gamma_{N}}(\Omega)$ and $q \in \mathrm{L}^{2}(\Omega)$ we have

$$
\begin{aligned}
\left\|\nu^{1 / 2} \nabla(u-\widetilde{u})\right\|_{0, \Omega} & \leq \nu_{\ominus}^{-1 / 2} c_{\mathrm{FP}}\left(\Omega, \Gamma_{D}\right)\|\operatorname{div} \tau+f\|_{1, \ln , \Omega} \\
& +\left\|\nu^{-1 / 2}(\tau+q \mathbb{I}-\nu \nabla \widetilde{u})\right\|_{0, \Omega}+2 \nu_{\oplus}^{1 / 2} \kappa\left(\Omega, \Gamma_{D}\right)\|\operatorname{div} \widetilde{u}\|_{0, \Omega} .
\end{aligned}
$$


As in the case of $d \geq 3$, the estimate is simplified if $\operatorname{div} \widetilde{v}=0$ in $\mathbb{R}^{2} \backslash \bar{B}_{r_{2}}$ and additionally (if $\Gamma_{D}=\Gamma$ ) condition (38) is satisfied. Then, in Theorem 4.14 we can replace the constant $\kappa\left(\Omega, \Gamma_{D}\right)$ by $\kappa\left(\omega, \gamma_{D}\right)$. If the approximation $\widetilde{u}$ is solenoidal in $\Omega$, then the last term vanishes and we arrive at estimates similar to Theorem 4.1. They possess the same property: the upper bound coincides with the norm of the error on the left-hand side if $\tau=\sigma$ and $p=q$ (i.e., the estimate is sharp in the sense that there is no "gap" between its left- and right-hand sides).

For the approximation of the pressure function, Theorem 4.4 is modified as follows.

Theorem 4.15. Let $\tilde{p} \in \mathrm{L}^{2}(\Omega)$. Then for all $\tau \in \mathbb{D}_{\Gamma_{N}}(\Omega)$ and all $\widetilde{u} \in u_{D}+\mathrm{H}_{-1, \ln , \Gamma_{D}}^{1}(\Omega)$ we have

$$
\begin{aligned}
\|p-\widetilde{p}\|_{0, \Omega} & \leq \kappa\left(\Omega, \Gamma_{D}\right)\left(\left(\nu_{\ominus}^{-1 / 2} \nu_{\oplus}^{1 / 2}+1\right) c_{\mathrm{FP}}\left(\Omega, \Gamma_{D}\right)\|\operatorname{div} \tau+f\|_{1, \ln , \Omega}\right. \\
& \left.+2 \nu_{\oplus}^{1 / 2}\left\|\nu^{-1 / 2}(\tau+\widetilde{p} \mathbb{I}-\nu \nabla \widetilde{u})\right\|_{0, \Omega}+2 \nu_{\oplus} \kappa\left(\Omega, \Gamma_{D}\right)\|\operatorname{div} \widetilde{u}\|_{0, \Omega}\right)
\end{aligned}
$$

Finally, we consider a nonconforming approximation $\widetilde{u}$ and obtain analogs of the theorems exposed in Subsection 4.3.

Theorem 4.16. Suppose that $\widetilde{\Upsilon} \in \mathrm{L}^{2}(\Omega)$ and $\tilde{p} \in \mathrm{L}^{2}(\Omega)$. Then for all $\widetilde{u} \in u_{D}+$ $\mathrm{H}_{-1, \ln , \Gamma_{D}}^{1}(\Omega), \tau \in \mathbb{D}_{\Gamma_{N}}(\Omega)$, and $q \in \mathrm{L}^{2}(\Omega)$ we have

$$
\begin{aligned}
\left\|\nu^{1 / 2}(\Upsilon-\widetilde{\Upsilon})\right\|_{0, \Omega} \leq & \nu_{\ominus}^{-1 / 2} c_{\mathrm{FP}}\left(\Omega, \Gamma_{D}\right)\|\operatorname{div} \tau+f\|_{1, \ln , \Omega}+\left\|\nu^{-1 / 2}(\tau+q \mathbb{I}-\nu \nabla \widetilde{u})\right\|_{0, \Omega} \\
& +2 \nu_{\oplus}^{1 / 2} \kappa\left(\Omega, \Gamma_{D}\right)\|\operatorname{div} \widetilde{u}\|_{0, \Omega}+\left\|\nu^{1 / 2}(\nabla \widetilde{u}-\widetilde{\Upsilon})\right\|_{0, \Omega} \\
\leq & \nu_{\ominus}^{-1 / 2} c_{\mathrm{FP}}\left(\Omega, \Gamma_{D}\right)\|\operatorname{div} \tau+f\|_{1, \ln , \Omega}+\left\|\nu^{-1 / 2}(\tau+q \mathbb{I}-\nu \widetilde{\Upsilon})\right\|_{0, \Omega} \\
& +2 \nu_{\oplus}^{1 / 2} \kappa\left(\Omega, \Gamma_{D}\right)\|\operatorname{div} \widetilde{u}\|_{0, \Omega}+2\left\|\nu^{1 / 2}(\nabla \widetilde{u}-\widetilde{\Upsilon})\right\|_{0, \Omega}
\end{aligned}
$$

and

$$
\begin{gathered}
\|p-\widetilde{p}\|_{0, \Omega} \leq \kappa\left(\Omega, \Gamma_{D}\right)\left(\left(\nu_{\ominus}^{-1 / 2} \nu_{\oplus}^{1 / 2}+1\right) c_{\mathrm{FP}}\left(\Omega, \Gamma_{D}\right)\|\operatorname{div} \tau+f\|_{1, \ln , \Omega}\right. \\
+2 \nu_{\oplus}^{1 / 2}\left\|\nu^{-1 / 2}(\tau+\widetilde{p} \mathbb{I}-\nu \widetilde{\Upsilon})\right\|_{0, \Omega}+2 \nu_{\oplus} \kappa\left(\Omega, \Gamma_{D}\right)\|\operatorname{div} \widetilde{u}\|_{0, \Omega} \\
\left.+2 \nu_{\oplus}^{1 / 2}\left\|\nu^{1 / 2}(\nabla \widetilde{u}-\widetilde{\Upsilon})\right\|_{0, \Omega}\right) .
\end{gathered}
$$

For $\widetilde{\Upsilon}=\nabla \widetilde{u}$, where $\widetilde{u} \in u_{D}+\mathrm{H}_{-1, \ln , \Gamma_{D}}^{1}(\Omega)$, we recover Theorem 4.14 and Theorem 4.15. As in Subsection 4.4, error estimates for the stress tensor field $\sigma$ follow immediately by the triangle inequality.

Finally, we briefly present lower bounds of the error derived in the spirit of Subsection 4.5 .

Theorem 4.17. For all $\tilde{u}, v \in u_{D}+\mathrm{H}_{-1, \ln , \Gamma_{D}}^{1}(\Omega)$ and all $\varphi \in \mathrm{H}_{-1, \ln , \Gamma_{D}}^{1}(\Omega), \tau \in \mathbb{D}_{\Gamma_{N}}(\Omega)$, and $q \in \mathrm{L}^{2}(\Omega)$ we have

$$
\begin{aligned}
\left\|\nu^{1 / 2} \nabla(u-\widetilde{u})\right\|_{0, \Omega}^{2} & \geq 2\langle f, \varphi\rangle_{0, \Omega}+2\langle q, \operatorname{div} \varphi\rangle_{0, \Omega}-\langle\nu \nabla(2 \widetilde{u}+\varphi), \nabla \varphi\rangle_{0, \Omega} \\
& -2 \kappa\left(\Omega, \Gamma_{D}\right)\|\operatorname{div} \varphi\|_{0, \Omega}\left(\left(\nu_{\ominus}^{-1 / 2} \nu_{\oplus}^{1 / 2}+1\right) c_{\mathrm{FP}}\left(\Omega, \Gamma_{D}\right)\|\operatorname{div} \tau+f\|_{1, \ln , \Omega}\right. \\
& \left.+2 \nu_{\oplus}^{1 / 2}\left\|\nu^{-1 / 2}(\tau+q \mathbb{I}-\nu \nabla v)\right\|_{0, \Omega}+2 \nu_{\oplus} \kappa\left(\Omega, \Gamma_{D}\right)\|\operatorname{div} v\|_{0, \Omega}\right) .
\end{aligned}
$$

In particular, $v=\widetilde{u}$ is possible. If $\varphi \in \mathrm{S}_{-1, \ln , \Gamma_{D}}(\Omega)$, the estimate simplifies to

$$
\left\|\nu^{1 / 2} \nabla(u-\widetilde{u})\right\|_{0, \Omega}^{2} \geq 2\langle f, \varphi\rangle_{0, \Omega}-\langle\nu \nabla(2 \widetilde{u}+\varphi), \nabla \varphi\rangle_{0, \Omega}
$$

and equality occurs for $\varphi=u-\widetilde{u}$, provided that $\widetilde{u} \in u_{D}+\mathrm{S}_{-1, \ln , \Gamma_{D}}(\Omega)$. 
Finally, to handle nonconforming approximations $\widetilde{\Upsilon} \in \mathrm{L}^{2}(\Omega)$ we use again the triangle inequality to estimate

$$
\left\|\nu^{1 / 2}(\Upsilon-\widetilde{\Upsilon})\right\|_{0, \Omega} \geq\left\|\nu^{1 / 2} \nabla(u-\widetilde{u})\right\|_{0, \Omega}-\left\|\nu^{1 / 2}(\nabla \widetilde{u}-\widetilde{\Upsilon})\right\|_{0, \Omega}
$$

whenever $\widetilde{u} \in u_{D}+\mathrm{H}_{-1, \ln , \Gamma_{D}}^{1}(\Omega)$. Theorem 4.17 applied to the first term on the righthand side yields an estimate similar to Theorem 4.8.

\section{REFERENCES}

[1] I. Babuška and A. K. Aziz, Survey lectures on the mathematical foundations of the finite element method, Acad. Press, New York, 1972. MR 0421106 (54:9111)

[2] S. Bauer, D. Pauly, and M. Schomburg, The Maxwell compactness property in bounded weak Lipschitz domains with mixed boundary conditions, SIAM J. Math. Anal. 48 (2016), no. 4, 2912-2943. MR 3542004

[3] F. Brezzi, On the existence, uniqueness and approximation of saddle-point problems arising from Lagrange multipliers, Rev. Francaise Automat. Informat. Recherche Operationnelle Ser. Rouge 8 (1974), no. R-2, 129-151. MR 0365287 (51:1540)

[4] S. Cochez-Dhondt, S. Nicaise, and S. Repin, A posteriori error estimates for finite volume approximations, Math. Model. Nat. Phenom. 4 (2009), no. 1, 106-122. MR 2483555 (2009m:65202)

[5] M. Costabel, A remark on the regularity of solutions of Maxwell's equations on Lipschitz domains, Math. Methods Appl. Sci. 12 (1990), no. 4, 365-368. MR 1048563 (91c:35028)

[6] M. Costabel and M. Dauge, On the inequalities of Babuskä-Aziz, Friedrichs and Horgan-Payne, arXiv $1303.6141 \mathrm{v} 1,2013$

[7] M. Fuchs and S. Repin, Estimates of the deviations from the exact solutions for variational inequalities describing the stationary flow of certain viscous incompressible fluids, Math. Methods Appl. Sci. 33 (2010), no. 9, 1136-1147. MR 2668900 (2011f:76032)

[8] G. Galdi, An introduction to the mathematical theory of the Navier-Stokes equations. Vol. 1, Springer Tracts Natur. Philos., vol. 38, Springer, New York, 1994. MR 1284205 (95i:35216a)

[9] V. Girault and A. Sequeira, A well-posed problem for the exterior Stokes equations in two and three dimensions, Arch. Rational Mech. Anal. 114 (1991), no. 4, 313-333. MR 1100798 (92c:35093)

[10] C. Horgan and L. Payne, On inequalities of Korn, Friedrichs and Babuskä-Aziz, Arch. Ration. Mech. Anal. 82 (1983), no. 2, 165-179. MR 0687553 (84d:73014)

[11] M. Kessler, Die Ladyzhenskaya-Konstante in der numerischen Behandlung von Strömungsproblemen, Dissertat. Doktorgrades, Bayerischen Julius-Maximilians-Universität, Würzburg, 2000.

[12] P. Kuhn and D. Pauly, Regularity results for generalized electro-magnetic problems, Analysis (Munich) 30 (2010), no. 3, 225-252. MR 2676208 (2011k:35227)

[13] O. A. Ladyzhenskaya, Mathematical problems in the dynamics of a viscous incompressible Flow, FIZMATLIT, M., 1961; English transl, Second ed., Gordon\&Breach, New York, 1969. MR 0155093 (27:5034b)

[14] O. A. Ladyzhenskaya and V. A. Solonnikov, Some problems of vector analysis, and generalized formulations of boundary value problems for the Navier-Stokes equation, Zap. Naucn. Sem. Leningrad. Otdel. Mat. Inst. Steklov. (LOMI) 59 (1976), 81-116; English transl., J. Soviet Math. 10(1978), no. 2, 257-286. MR 0467031 (57:6900)

[15] R. Lazarov, S. Repin, and S. Tomar, Functional a posteriori error estimates for discontinuous Galerkin approximations of elliptic problems, Numer. Methods Partial Differential Equations 25 (2009), no. 4, 952-971. MR 2526991 (2010e:65195)

[16] R. Leis, Initial boundary value problems in mathematical physics, Teubner, Stuttgart, 1986. MR 0841971 (87h:35003)

[17] O. Mali, P. Neittaanmäki, and S. Repin, Accuracy verification methods. Theory and algorithms, Computat. Methods Appl. Sci., vol. 32, Springer, Dordrecht, 2014. MR 3136124

[18] A. Mikhaylov and S. Repin, Estimates of deviations from exact solution of the Stokes problem in the vorticity-velocity-pressure formulation, Zap. Naucn. Sem. S.-Peterburg. Otdel. Mat. Inst. Steklov. (POMI) 397 (2011), 73-88; English transl., J. Math. Sci. (N.Y.) 185 (2012), no. 5, 698-706. MR 2870109

[19] S. G. Mikhlin, Variational methods in mathematical physics, GITTL, M., 1957; English transl., Pergamon Press, Oxford, 1964. MR 0172493 (30:2712)

[20] _ Constants in some inequalities of analysis, John Wiley \& Sons, Ltd., Chichester, 1986. MR 0853915 (87g:46057) 
[21] S. Nazarov and K. Pilecka, On steady Stokes and Navier-Stokes problems with zero velocity at infinity in a three-dimensional exterior domain, J. Math. Kyoto Univ. 40 (2000), no. 3, 475-492. MR 1794517 (2001j:35226)

[22] J. Nečas, Les méthodes directes en théorie des équations elliptiques, Masson et Cie, Éditeurs, Paris; Academia, Éditeurs, Prague, 1967. MR 0227584 (37:3168)

[23] P. Neittaanmäki and S. Repin, Reliable methods for computer simulation. Error control and a posteriori estimates, Stud. Math. Appl., vol. 33, Elsevier, Amsterdam, 2004. MR 2095603 (2005k:65005)

[24] A posteriori error majorants for approximations of the evolutionary Stokes problem, J. Numer. Math. 18 (2010), no. 2, 119-134. MR 2657641 (2011e:65167)

[25] M. A. Ol'shanskii and E. V. Chizhonkov, On the best constant in the inf-sup condition for elongated rectangular domains, Mat. Zametki 67 (2000), no. 3, 387-396; English transl., Math. Notes 67 (2000), no. 3-4, 325-332. MR 1779472 (2001g:76020)

[26] D. Pauly, Generalized electro-magneto statics in nonsmooth exterior domains, Analysis (Munich) 27 (2007) no. 4, 425-464. MR 2373665 (2009h:35420)

[27] D. Pauly and S. Repin, Functional a posteriori error estimates for elliptic problems in exterior domains, J. Math. Sci. (N.Y.) 162 (2009), no. 3, 393-406. MR 2839012 (2012k:35099)

[28] _ The stationary Stokes problem in exterior domains: estimates of the distance to solenoidal fields and functional a posteriori error estimates, https://arxiv.org/abs/1810.12555, 2018.

[29] L. E. Payne, A bound for the optimal constant in an inequality of Ladyzhenskaya and Solonnikov, IMA J. Appl. Math. 72 (2007), no. 5, 63-569. MR 2361570 (2008k:35362)

[30] R. Picard, Zur Theorie der harmonischen Differentialformen, Manuscripta Math. 1 (1979), no. 1, 31-45. MR 0524976 (80m:58002)

[31] _ Randwertaufgaben der verallgemeinerten Potentialtheorie, Math. Methods Appl. Sci. 3 1981, no. 2, 218-228. MR 0657293 (83h:31007)

[32] - On the boundary value problems of electro- and magnetostatics, Proc. Roy. Soc. Edinburgh Sect. A 92 (1982), no. 1-2, 165-174. MR 0667134 (83m:78006)

[33] W. Prager and J. L. Synge, Approximation in elasticity based on the concept of function space, Quart. Appl. Math. 5 (1947), 241-269. MR 0025902 (10:81b)

[34] S. Repin, A posteriori estimation for variational problems with uniformly convex functionals, Math. Comput. 69 (2000), no. 230, 481-500. MR 1681096 (2000i:49046)

[35] — A posteriori estimates for the Stokes problem, Zap. Nauchn. Sem. S.-Peterburg. Otdel. Mat. Inst. Steklov. (POMI) 259(1999), 195-211, English transl., J. Math. Sci. (N. Y.) 109 (2002), no. 5, 1950-1964. MR 1754364 (2001c:35193)

[36] _ Estimates of deviation from the exact solutions for some boundary-value problems with incompressibilily condition, Algebra i Analiz 16 (2004), no. 5, 124-161; English transl., St. Petersburg Math. J. 16 (2005), no. 5, 837-862. MR 2106670 (2005i:35213)

[37] _ A posteriori estimates for partial differential equations, Radon Ser. Comput. Appl. Math., vol. 4, Walter de Gruyter, Berlin, 2008. MR 2458008 (2010b:35004)

[38] _ Estimates of the distance to the set of divergence free fields, Zap. Nauchn. Sem. S.Peterburg. Otdel. Mat. Inst. Steklov. (POMI) 425 (2014), 99-116; English transl., J. Math. Sci. (N.Y.) 210 (2015), no. 6, 822-834. MR 3407795

[39] _ Estimates of the distance to the set of solenoidal vector fields and applications to a posteriori error control, Comput. Methods Appl. Math. 15 (2015), no. 4, 515-530. MR 3407790

[40] _ Localized forms of the LBB condition and a posteriori estimates for incompressible media problems, Math. Comput. Simulation 145 (2018), 156-170. MR 3763528

[41] S. Repin and R. Stenberg, A posteriori estimates for a generalized Stokes problem, Zap. Nauchn. Sem. S.-Peterburg. Otdel. Mat. Inst. Steklov. (POMI) 362 (2008), 272-302; English transl., J. Math. Sci. (N.Y.) 159 (2009), no. 4, 541-558. MR 2760557

[42] S. Repin, S. Sysala, and J. Haslinger, Computable majorants of the limit load in Henckys plasticity problems, Comput. Math. Appl. 75 (2018), 199-217. MR 3758699

[43] J. Saranen and K.-J. Witsch, Exterior boundary value problems for elliptic equations, Ann. Acad. Sci. Fenn. Ser. Math. 8 (1983), no. 1, 3-42. MR 0698835 (84f:35043)

[44] G. Stoyan, Towards discrete Velte decompositions and narrow bounds for Inf-Sup constants, Comput. Math. Appl. 38 (1999), no. 7-8, 243-261. MR 1713178 (2000k:65154)

[45] S. Tomar and S. Repin, Efficient computable error bounds for discontinuous Galerkin approximations of elliptic problems, J. Comput. Appl. Math. 226 (2009), no. 2, 358-369. MR 2502931 (2010d:65335)

[46] R. Verfürth, A posteriori error estimators for the Stokes equations, Numer. Math. 55 (1989), no. 3, 309-326. MR 0993474 (90d:65187) 
[47] _ A review of a posteriori error estimation and adaptive mesh-refinement techniques, WileyTeubner, Stuttgart, 1996.

[48] H. Weyl, The method of orthogonal projections in potential theory, Duke. Math. J. 7 (1940), 411444. MR 0003331 (2:202a)

Fakultät für Mathematik, Universität Duisburg-Essen, Campus Essen, Germany; Faculty of Information Technology, University of Jyväskylä, Finland

Email address: dirk.pauly@uni-due.de

Steklov Institute of Mathematics, St.Petersburg, Russia; Peter the Great St. Petersburg Polytechnic University, St. Petersburg, Russia; Faculty of Information Technology, UNIVERSity OF JyVÄSKYlä, Finland

Email address: repin@pdmi.ras.ru

Received 1/NOV/2018

Originally published in English 